\title{
Los mapas conceptuales como instrumento de evaluación: Una experiencia de educación ambiental centrada en el estudio de ecosistemas acuáticos
}

Conceptual maps as an evaluation instrument: an experience in environmental education through the study of aquatic ecosystems

\author{
Volumen 18, Número 1 \\ Enero-Abril \\ pp. 1-35
}

Este número se publica el $1^{\circ}$ de enero de 2018

DOI: https://doi.org/10.15517/aie.v18i1.31840

Laura Galván Pérez

José Gutiérrez Pérez

Revista indizada en REDALYC, $\underline{\text { SCIELO }}$

Revista distribuida en las bases de datos:

LATINDEX, DOAJ, REDIB, IRESIE, CLASE, DIALNET, SHERPA/ROMEO, QUALIS-CAPES, MIAR

Revista registrada en los directorios:

ULRICH'S $, \underline{\text { REDIE}}, \underline{\text { RINACE}}, \underline{\text { OEI }}$ MAESTROTECA, PREAL, $\underline{\text { CLACSO }}$ 


\title{
Los mapas conceptuales como instrumento de evaluación: Una experiencia de educación ambiental centrada en el estudio de ecosistemas acuáticos
}

Conceptual maps as an evaluation instrument: an experience in environmental education through the study of aquatic ecosystems

\author{
Laura Galván Pérez ${ }^{1}$ \\ José Gutiérrez Pérez²
}

\begin{abstract}
Resumen. Los ecosistemas acuáticos generan una serie de servicios fundamentales para mantener el bienestar humano y el mantenimiento de la vida en la biosfera. Sin embargo, la actividad humana está generando un gran impacto en dichos ecosistemas, pues altera el funcionamiento y el mantenimiento de estos, así como pone en riesgo la supervivencia y el bienestar humano. Para ello, es fundamental determinar si los diferentes recursos didácticos que trabajan la temática agua en la educación formal están permitiendo generar dicho conocimiento y una conciencia social ambiental sobre dicha cuestión. El artículo tiene como objeto diseñar y aplicar un modelo de evaluación de mapas conceptuales a una muestra de 23 estudiantes de tercer grado de Educación Secundaria Obligatoria en Huelva, España, con el fin de detectar, por un lado, las ideas previas, y por otro, identificar si se produjo aprendizaje conceptual y actitudinal en torno al agua tras el desarrollo de un recurso educativo. Para ello, se llevó a cabo un plan de intervención en el aula, de carácter mixto, consistente en 3 sesiones de trabajo. Se utilizaron, además los mapas conceptuales como técnica de recolección de datos. Los resultados de la investigación señalan que el análisis de cada uno de los elementos de los mapas conceptuales (número de conceptos, número de conectores, nivel de jerarquía, impacto visual y las relaciones entre los conceptos), aumentó después de la aplicación del recurso didáctico. En conclusión, el recurso educativo favoreció el aprendizaje conceptual y actitudinal en torno la importancia del recurso hídrico de los ecosistemas acuáticos agua, con lo cual se obtuvieron mejores niveles de aprendizaje.
\end{abstract}

Palabras clave: mapas conceptuales, educación ambiental, agua, recursos educativos.

\begin{abstract}
The aquatic ecosystems generate a number of essential services for maintaining human well-being and maintaining life in the biosphere. However, human activity is having a great impact on these ecosystems, altering the operation and maintenance of them, as well as putting at risk the survival and the human well-being. For this, it is fundamental to determine if the different didactic resources that work the theme water in the formal education, are allowing to generate such knowledge and an environmental social conscience. The objective of this article is to design and apply a conceptual map evaluation model to a sample of 23 students from 3rd level of Compulsory Secondary Education, in order to detect, on the one hand, the previous ideas, and on the other hand, the identification of conceptual and attitudinal learning about water, following the development of an educational resource. For this purpose, a mixed classroom intervention plan was carried out, consisting of 3 work sessions, using conceptual maps as a data collection technique. The results of the research indicate that the analysis of each element of the conceptual maps (number of concepts, number of connectors, level of hierarchy, visual impact and relations between concepts) increased after the application of the didactic resource. In conclusion, the educational resource fosters the conceptual and attitudinal learning about water, obtaining better levels of learning.
\end{abstract}

Keywords: conceptual maps, environmental education, water, educational resources.

\footnotetext{
${ }^{1}$ Doctoranda del Programa de Ciencias de la Educación, Universidad de Granada, España.Dirección electrónica: lauragp@correo.ugr.es

${ }^{2}$ Catedrático de Métodos de Investigación y Diagnóstico en Educación, Universidad de Granada, España.Dirección electrónica: iguti@ugr.es
}

Artículo recibido: 29 de abril, 2017

Enviado a corrección: 01 de noviembre, 2017

Aprobado: 04 de diciembre, 2017 


\section{Introducción}

La construcción del conocimiento científico y ambiental es producto de la interacción del estudiantado con su entorno, con sus iguales y con los recursos que promueven y motivan la búsqueda de respuestas significativas que contribuyen a una progresiva abstracción reflexiva, a una búsqueda de regularidades, reglas, patrones y relaciones que favorecen la representación mental de la realidad en forma de construcciones conceptuales y redes de relaciones. De ahí que sean de gran interés los recursos educativos que favorezcan estos aprendizajes como recursos mediadores de los procesos de aprendizaje significativo.

Una definición clásica de recurso didáctico consiste en: "Aquellos medios materiales de que se dispone para conducir el aprendizaje de los alumnos" (Mattos, 1963, p. 34). Una definición más actual incluye facetas nuevas, que ponen énfasis en los soportes tecnológicos, más allá de los objetos, artefactos, materiales físicos, maquetas y modelos manipulativos para abrir paso a los medios audiovisuales y los instrumentos didácticos virtuales de nueva generación, apoyados en portales interactivos y realidad aumentada (Anta, 2010; Fracchia, Armiño y Martins, 2015; López y Morcillo, 2007; Moreno, 2014; Sabbatini, 2004).

Prácticamente, en casi todas las situaciones de enseñanza presencial o virtual se emplean recursos didácticos de todo tipo y en diferentes tipos de soporte, los cuales condicionan el proceso de aprendizaje y contribuyen de una u otra forma a estimularlo con mayor o menor éxito (Ojeda, Gutiérrez y Perales, 2011).

Uno de los recursos educativos frecuentemente utilizados es el uso del vídeo con fines didácticos, el cual tiene un gran potencial educativo, y su utilización en las aulas constituye una excelente vía para el logro de aprendizajes significativos. Algunas de sus ventajas son: versatilidad, medio expresivo, medio motivacional, mejor acceso a los significados (imagenpalabra-sonido), entre otras (Moreno, 2014). Entre las desventajas, se destacan: la elección del recurso adecuado a la finalidad educativa; la complejidad lingüística, la cual puede exceder el nivel de comprensión del alumno; la falta de interés en el alumnado de lo que se está visualizando y también la sensación de pasividad en el mismo.

El medio audiovisual es una herramienta curricular de muy fácil acceso en nuestros días, de uso bastante frecuente en las aulas y cuyo empleo al servicio de la sensibilización, concienciación y educación ambiental se ha incrementado gracias a la variedad de propuestas de acceso abierto al alcance de la persona docente. Para el caso que nos ocupa, estudio de ecosistemas acuáticos, podemos encontrar copiosas referencias sobre esta 
temática ${ }^{3}$ hasta un total de 72.500 resultados sobre "vídeos educativos sobre el agua"; 94.900 sobre "El ciclo del agua" y 97.800 sobre la contaminación del agua 4 . El problema que ha de afrontar el profesorado ante tal volumen y variedad de recursos consiste en llevar a cabo una evaluación adecuada que permita la selección de material de suficiente calidad pedagógica, apropiado para las edades de los escolares y coherente con las finalidades y objetivos curriculares propuestos (Galván y Gutiérrez, 2017). No obstante, el uso de los recursos y medios audiovisuales por sí mismos no contribuye de forma espontánea al desarrollo de competencias conceptuales y actitudinales que mejoren los niveles de alfabetización ambiental de los y las escolares.

Los recursos audiovisuales a emplear, además de cumplir unos criterios de calidad mínimos, han de estar integrados en una propuesta metodológica que contribuya a su eficacia y eficiencia curricular. En nuestro caso, acompañamos el uso de recursos audiovisuales con una metodología de trabajo de aula, basada en el empleo de mapas conceptuales como instrumentos de autorregulación y control del propio aprendizaje por parte del estudiantado, y como parte de un instrumento de evaluación por la persona docente dado que el mapa conceptual es una herramienta que permite representar la estructura de conceptos de una temática concreta (Fernández y Rodríguez, 1995).

Los mapas conceptuales constituyen un instrumento útil de representación del conocimiento, el cual ayuda a reconocer visualmente los conceptos más importantes, las relaciones entre ellos y la forma de organización jerárquica en grados de dificultad o de importancia (González, 2008; Novak y Gowin, 1988). En este sentido, los mapas conceptuales favorecen el aprendizaje significativo al permitir modificar la estructura cognitiva mediante nuevos conocimientos relacionados con los anteriormente adquiridos, para ello es fundamental la motivación del alumnado en su elaboración y uso (Pozo, 1989). En definitiva, los mapas conceptuales son una técnica que se utiliza para obtener una representación visual de las ideas de una persona sobre un concepto o un conjunto de conceptos relacionados. Asimismo, hay evidencias empíricas de que los mapas conceptuales fomentan la reflexión, favorecen la comprensión y la diferenciación de conceptos (Pontes, Serrano y Muñoz, 2015; Pontes, 2012; Valadares, 2013). Esta comprensión significativa, que se va logrando al construir mapas conceptuales, favorece, igualmente, procesos de tipo metacognitivos, los cuales permiten al estudiante tomar

3 El Portal de UNESCO incluye un amplio listado de recursos de alta calidad educativa sobre el tópico: http://www.unesco.org/archives/multimedia/?page=4\&pg=34\&kw=Water+resources

4 Información recuperada por medio de Youtube durante noviembre del 2017. 
conciencia sobre su propio aprendizaje (Castaño, 2010; Prietro y Chrobak, 2013). También, son útiles para identificar las ideas previas del alumnado (Molina-Azcárate, 2013; Rivadulla, García y Martínez, 2015), así como instrumentos para evaluar el aprendizaje y el desarrollo cognitivo (Correia, Silva y Junior, 2010), permitiendo determinar el grado en que los objetivos de importancia educativa, están siendo alcanzados, en la medida en que la evaluación consiste en la formulación de un juicio de valor o de mérito, para apreciar los resultados educativos en términos de si están satisfaciendo o no, un conjunto específico de metas curriculares (Ausubel, Novak y Hanesian, 1989).

En este sentido, parece pertinente utilizar los mapas conceptuales como detectores de ideas previas, pues con ellos se determinará el punto de partida. Resulta importante también, su consideración como instrumentos de evaluación que permitan determinar si tras la aplicación de un recurso educativo concreto se produjo un aprendizaje conceptual y actitudinal en torno al agua. Por ello, se plantea la necesidad de desarrollar el diseño de un modelo metodológico de evaluación de los mapas conceptuales.

Por lo tanto, los objetivos concretos de la presente investigación son:

A. Detectar las ideas previas del alumnado sobre la contaminación del agua a través de mapas conceptuales.

B. Diseñar un instrumento de evaluación para determinar el nivel de aprendizaje conceptual y actitudinal adquirido en torno a la temática del agua tras la aplicación y desarrollo de un recurso didáctico.

\section{El marco teórico}

\subsection{Aprendizaje significativo y educación ambiental: Mapas conceptuales}

La teoría del aprendizaje significativo (Ausubel, 1968; Ausubel, Novak y Hanesian, 1989) establece que el aprendizaje se produce cuando la persona es capaz de establecer relaciones significativas y no arbitrarias entre el nuevo contenido y su conocimiento previo. Esta asimilación ocurre en función de las relaciones jerárquicas que el individuo establece entre los conceptos. En dichas relaciones el concepto más inclusivo asimila otros conceptos más específicos; de manera que, en este proceso, todos los conceptos van adquiriendo un nuevo significado para el individuo. Cuanto más substanciales sean las relaciones que un individuo establece entre su conocimiento previo y la nueva información que recibe, tanto más significativo será su proceso de aprendizaje (Guruceaga y González, 2004). 
En otro orden de ideas, la teoría de la educación de Novak (1977, 1990, 1998), que continúa y desarrolla la teoría del aprendizaje significativo, (Ausubel, 1968), propone como una de las técnicas de enseñanza el uso de mapas conceptuales durante el proceso de enseñanza (Ausubel et al., 1989; Echarri y Puig, 2008). En este sentido, según Ballester (2002), el mapa conceptual es uno de los instrumentos más adecuados para promover aprendizajes significativos, ya que en él los conceptos presentados están conectados con una coherencia interna y una conexión adecuada.

En efecto, los mapas conceptuales son una red de proposiciones entre conceptos que pueden estar unidos mediante frases breves o palabras de enlace que evidencian el significado de la relación conceptual, indican el nivel y la complejidad de la comprensión y permiten representar el conocimiento que tiene una persona sobre un tema. Dichas proposiciones están organizadas en el mapa, se relacionan gráficamente y forman cadenas o unidades semánticas que poseen significado. Esta representación del conocimiento ayuda a reconocer visualmente los conceptos más importantes del tema, las relaciones que se establecen entre ellos, su forma de organización jerárquica y permiten construir una imagen mental de la información que estamos procesando (Rivaduella, García y Martínez, 2015).

Por lo tanto, la elaboración de un mapa conceptual requiere: identificar los principales conceptos, ordenar estos de lo general a lo específico (los conceptos más generales quedan en la parte superior y los más específicos, dispuestos jerárquicamente abajo), y por último, establecer líneas que reflejen las relaciones de mayor relevancia y que unan los diferentes conceptos entre sí (Fernández y Rodríguez, 1995).

En definitiva, los mapas conceptuales son una herramienta pedagógica de representación del conocimiento (Novak, 1998), utilizados para promover un aprendizaje más significativo que contribuye a sistematizar y estructurar la información. En este sentido, según dicho autor, el aprendizaje será más significativo cuando se contemplen las siguientes ideas: a.) el aprendizaje significativo se produce más fácilmente cuando los nuevos significados conceptuales se engloban bajo otros conceptos más amplios o inclusivos; b.) los conceptos en la estructura cognitiva sufren una diferenciación progresiva que hace que se puedan reconocer más vínculos proposicionales con otros conceptos, y c.) cuando dos o más conceptos se relacionan en términos de nuevos significados proposicionales tiene lugar la reconciliación integradora (Fernández y Rodríguez, 1995).

En este sentido, a la hora de analizar el potencial educativo de los mapas conceptuales, como favorecedores de aprendizaje significativo, pueden establecerse una 
serie de ítems a reconocer en los mapas, y así determinar el nivel de aprendizaje. Algunos de estos ítems pueden ser: qué conceptos utiliza y cuáles no; qué proposiciones erróneas establece; cómo estas proposiciones están estrechamente vinculadas a jerarquías conceptuales no lógicas (González, Morón y Novak, 2001); cuál es el concepto que el estudiante considera más inclusivo, y a través de qué diferenciaciones progresivas le atribuye significado; y por último, también se presenta interesante reconocer las relaciones sin jerarquía entre conceptos diferenciados a través de las denominadas reconciliaciones integradoras, las cuales pueden considerarse como el factor más creativo (Guruceaga y González, 2004).

Por último, el aprendizaje será significativo, cuando sea capaz de despertar la motivación y el interés del alumnado, siendo un factor clave la temática que se presente, y en este sentido, siendo un posible aliado para ello, las temáticas relacionadas con la problemática ambiental, por el desafío que suponen en las sociedades contemporáneas. De hecho, Novak (1978) plantea una relación entre la educación ambiental y su teoría de la educación, al presentarla como una herramienta pedagógica que permite promover los conocimientos, destrezas, valores y actitudes planteados por la educación ambiental. El autor destaca el importante papel del estudiante al responsabilizarse de su propio proceso pedagógico. Asimismo y enfatiza en el factor emocional como elemento que potencia aprendizajes más significativos, con lo cual se facilita un cambio en las actitudes necesarias para los desafíos planteados ante la problemática ambiental actual. En palabras de Gowin (1981), se posibilita desarrollar una educación que integre pensamiento, sentimiento y acción. Esta última "pasar a la acción", es un factor clave para los cambios necesarios en los estilos de vida y modos de desarrollo más acorde a una sociedad más justa y sostenible.

\subsection{La mirada social del agua}

Bajo la perspectiva del paradigma reduccionista, el agua es frecuentemente considerada como un simple recurso a consumir, gestionado como un tipo de fluido cautivo y menospreciado, sometido a canalización, comercio y mercantilización. Sin embargo, el agua desde la mirada de la complejidad es un elemento natural bastante más poliédrico y escurridizo, heterodoxo y versátil a nivel conceptual y funcional, ya sea por el lugar que ocupa su ciclo en la regulación de la habitabilidad del planeta, ya sea por la heterogeneidad de servicios que le brinda a nuestra civilización su presencia en los diferentes ecosistemas 
acuáticos: "[...] nada vivo es ajeno a las capacidades, destrezas, servicios y acogidas que el agua pone a disposición de todos [...]" (Araujo, 2012, p. 4).

Si se parte de la idea del agua como elemento natural aislado y descontextualizado de su ecosistema, implícitamente se está promoviendo un modelo parcial de conceptualización sesgado y mermado hacia el uso y la gestión del recurso, hecho que lo pone en peligro al ignorar su contexto y reducir su esencia y funcionalidad al mero valor de uso. Bajo esta concepción antropocéntrica del agua, estamos ignorando su infinito valor ecológico como elemento que interactúa en su contexto, que regula, riega, fertiliza y garantiza un amplio conjunto de prestaciones intransferibles. Al privar al ecosistema del agua, sin prever implicaciones y consecuencias globales, estamos degradándolo, mermando su habilidad depuradora y menoscabando la capacidad de resiliencia de este para proveer agua y brindar servicios fundamentales.

Los ecosistemas acuáticos, junto al resto de ecosistemas, son capaces de generar una serie de servicios diferenciados: de abastecimiento, procedentes de la estructura biótica y geótica (agua dulce, madera, medicinas); de regulación, vinculadas al buen funcionamiento de los ciclos e interacciones (climáticas, hídricas, de la calidad del aire,...); y culturales, asociadas a valores intangibles comunitarios (identidad cultural, saberes locales, ocio y recreación), de los cuales depende el mantenimiento de la vida en el planeta en sus múltiples manifestaciones y del bienestar humano en sus diferentes formas de concebirlo.

Gestionar el agua, como recurso enfocado a usos humanos exclusivamente, responde a un modelo miope de entender los sistemas naturales, lo cual conlleva a una degradación del ecosistema a corto y mediano plazo, poniendo en riesgo no solo el abastecimiento de agua, sino también una serie de servicios básicos para el desarrollo comunitario de nuestros pueblos.

Es fundamental cambiar el paradigma a través del cual se estudia el agua, para concebirlo no solo como un recurso, sino como un caudal de complejidades, y como un sistema dinámico, dotado de vida e interacciones. Así como, encaminar la gestión, hacia la perspectiva de la gestión integral de ese caudal de complejidades, priorizando la conservación de la estructura y el mantenimiento del buen funcionamiento de los mismos, e incluyendo una mirada holística en su gestión. Las propuestas de Bronfrenbrenner sobre las ecologías del desarrollo humano y el papel del ambiente refuerzan esta visión cíclica y dialógica de las interacciones entre el ambiente y la educación: 
El desarrollo humano es el proceso por el cual la persona en desarrollo adquiere una concepción del ambiente ecológico más amplia, diferenciada y válida, y se motiva y se vuelve capaz de realizar actividades que revelen las propiedades de ese ambiente, lo apoyen y lo reestructuren, a niveles de igual o mayor complejidad, en cuanto a su forma y su contenido (Bronfenbrenner, 1987, p. 47).

La educación para la sostenibilidad debe favorecer el cambio de paradigma y una orientación hacia un planteamiento de nuevos modelos de desarrollo y estilos de vida que caminen hacia un mundo más justo, dotado de mayor equidad social y preocupado por un mayor equilibrio ecológico. En este contexto, todo lo relacionado con el agua constituye un caudal de aprendizajes hacia este modelo holístico e integral de los recursos naturales.

\subsection{El agua en la educación formal}

La enseñanza de primaria, secundaria y bachillerato incluye áreas de conocimiento relacionadas con el agua; en educación primaria, en el área de conocimiento del medio; en la educación secundaria obligatoria, en ciencias sociales y ciencias de la naturaleza; y en bachillerato, en ciencias del mundo contemporáneo y cultura científica.

Los libros de texto constituyen uno de los recursos principales para analizar el tratamiento dado a estas temáticas. Un estudio realizado por la Universidad de Zaragoza (Martínez y Antoraz, 2002) llevó a cabo un análisis de los libros de texto en el que concluye que estos reflejan los mismos tópicos, los mismos errores conceptuales, los mismos valores y las mismas omisiones relevantes respecto al agua, que se promueve en los medios de comunicación, y en definitiva en la sociedad en general.

En relación con las percepciones del alumnado sobre el agua, una investigación dirigida por Marcén (2006) concluyó lo siguiente:

- La imagen construida por el alumnado acerca del agua es poco variada, pues se construye en torno a un número muy limitado de ideas, sobre todo en educación primaria.

- Una buena parte de las ideas sostienen conceptos muy estáticos, ligados a las propiedades del agua y a algunos factores ambientales.

- En contadas ocasiones se evidencia la comprensión de procesos, priman conceptos o datos sin elaborar. 
- Se potencia el tratamiento de hechos, sucesos y situaciones ligados al agua, que en general, no incitan al alumnado a cuestionar actuaciones personales o sociales.

Por último, un estudio llevado a cabo en un aula de Bachillerato sobre el análisis del proceso de construcción de conocimiento significativo, en relación con el uso, el consumo y la contaminación del agua, concluyó que la intervención didáctica planteada permitió que un número significativo de estudiantes transitara desde un pensamiento más simple hacia otro más complejo, resultaron claves los siguientes aspectos: ideas previas del alumnado, determinación de los contenidos según las preferencias del alumnado y el establecimiento de fases de inicio, desarrollo y cierre en cada actividad, e incluyendo también periodos de acción-reflexión, entre otros (Fernández y Solís, 2011).

\section{Metodología}

\subsection{El recurso educativo}

El recurso educativo utilizado en el proyecto de investigación fue un video educativo titulado La necesidad de depurar, destinado a edades comprendidas entre los 12 y 16 años y elaborado por la Fundación Centro de las Nuevas Tecnologías del Agua (CENTA) como herramienta de concienciación para la Campaña Agua Prestada, Devuélvela Depurada, perteneciente al Programa de Educación Ambiental para la Comunidad Educativa (ALDEA) de la Consejería de Medio Ambiente y Educación de la Junta de Andalucía ${ }^{5}$.

Por otra parte, en relación con el potencial educativo del recurso, este gira en torno a 7 ejes temáticos que son: Ciclo integral del agua (elementos y procesos); las actividades causantes de la contaminación del agua (domésticas, industriales); las sustancias contaminantes (detergente, aceite, sólidos en suspensión, entre otros); conceptos relacionados con la contaminación (agua residual, vertidos, agentes patógenos, entre otros); efectos y consecuencias de la contaminación (mal olor, suciedad, enfermedades, fin de la vida acuática, entre otros,...); proceso de depuración y por último, buenas prácticas (no arrojar basura al inodoro o al fregadero, uso racional del agua en el hogar; depuración de las aguas residuales y uso adecuado del detergente).

\footnotetext{
${ }^{5}$ El enlace al vídeo educativo fue recuperado de: https://www.youtube.com/watch?v=gUsOHG1MOZ4
} 


\subsection{Plan de intervención}

La muestra de investigación está conformada por un grupo de 23 estudiantes de $3^{\circ}$ de Educación Obligatoria Secundaria del IES Catedrático Pulido Rubio en Bonares, Huelva. En relación con el plan de intervención en el aula, este consistió en 3 sesiones de trabajo especificadas a continuación:

- Sesión 0. Presentación del proyecto de investigación: En esta sesión se presentó el equipo de investigación y el proyecto (contenido, sesiones de trabajo, metodología, etc); se realizó una explicación de cómo elaborar mapas conceptuales, presentándose un ejemplo de un mapa conceptual sobre la contaminación atmosférica, y finalmente se elaboró un mapa conceptual de otra temática diferente al proyecto.

- Sesión 1. Mapas conceptuales (PRE, previos): En esta primera sesión de trabajo se llevó a cabo la elaboración de mapas conceptuales por parte del alumnado, esto permitió identificar sus ideas y conocimientos previos con respecto a la temática; por lo tanto, determinó el punto de partida. La temática a tratar era la contaminación del agua, se introdujeron unos conceptos claves que sirvieran a modo orientativo en la confección del mapa (contaminación del agua, problemática socioambiental, actividad humana, entre otros), debiendo ser ampliados por conceptos propios del alumnado relacionados con la temática según los conceptos orientativos.

- Sesión 2. Aplicación y desarrollo del recurso: En esta sesión se llevó a cabo la proyección del vídeo educativo y posteriormente, de manera individual, se cumplimentó, por parte del alumnado, una ficha elaborada por el equipo de investigación, dirigida a favorecer el trabajo y síntesis de los contenidos claves y principales del vídeo (anexo 1. Ficha de trabajo).

- Sesión 3. Mapas conceptuales (POST, posteriores): En esta etapa se elaboraron nuevamente mapas conceptuales tras la aplicación del recurso educativo, con el objetivo de poder realizar una comparativa entre las ideas previas y la adquisición de las nuevas, mediante la evaluación de los mapas conceptuales pre y post. En esta última sesión, para la elaboración de los mapas post, se volvieron a presentar los mismos conceptos orientativos presentados en la sesión 1.

\subsection{El instrumento de evaluación}

Para la evaluación de los mapas conceptuales se han diseñado dos tipos de instrumentos de evaluación. El primero de ellos se caracteriza, en mayor medida, por su 
carácter cuantitativo. Se han tenido en cuenta una serie de criterios de valoración, los cuales han sido analizados por separado en los mapas conceptuales elaborados por el alumnado. La segunda propuesta metodológica se caracteriza por ser un instrumento más flexible, inclusivo y global, principalmente de carácter cualitativo. A continuación se detalla el diseño de cada uno de ellos.

En relación con el primer instrumento de evaluación, los criterios de valoración que se han tenido en cuenta han sido los siguientes: número de conceptos; número de conectores; nivel de jerarquía; relaciones entre los conceptos; impacto visual y calidad de los conceptos y de las frases. Los criterios de evaluación considerados parten de estudios anteriores planteados por varios autores (Fernández y Rodríguez, 1995; Miller et al., 2009; Rivadulla et al., 2015). Por otra parte, estos criterios de valoración llevan asociado un sistema de puntuación, de manera que han generado 3 escenarios de evaluación (bajo, medio, alto), como puede verse en la Tabla 1 y sus correspondientes ejemplos en las Figuras 1, 2 y 3.

Tabla 1. Criterios de valoración del primer instrumento de evaluación de mapas conceptuales

\begin{tabular}{|c|c|c|c|}
\hline $\begin{array}{c}\text { Criterios } \\
\end{array}$ & Nivel bajo & Nivel medio & Nivel alto \\
\hline Número de conceptos & $0-10$ & $11-20$ & $21-30$ \\
\hline Número de conectores & $0-15$ & $16-30$ & $31-50$ \\
\hline Nivel de jerarquía & $1-2$ & $3-4$ & 5 \\
\hline Impacto visual & Poco estructurado & Algo estructurado & Bastante estructurado \\
\hline
\end{tabular}

Fuente: elaboración propia (Galván Pérez, 2017)

Figura 1. Ejemplo de un mapa conceptual previo de nivel bajo

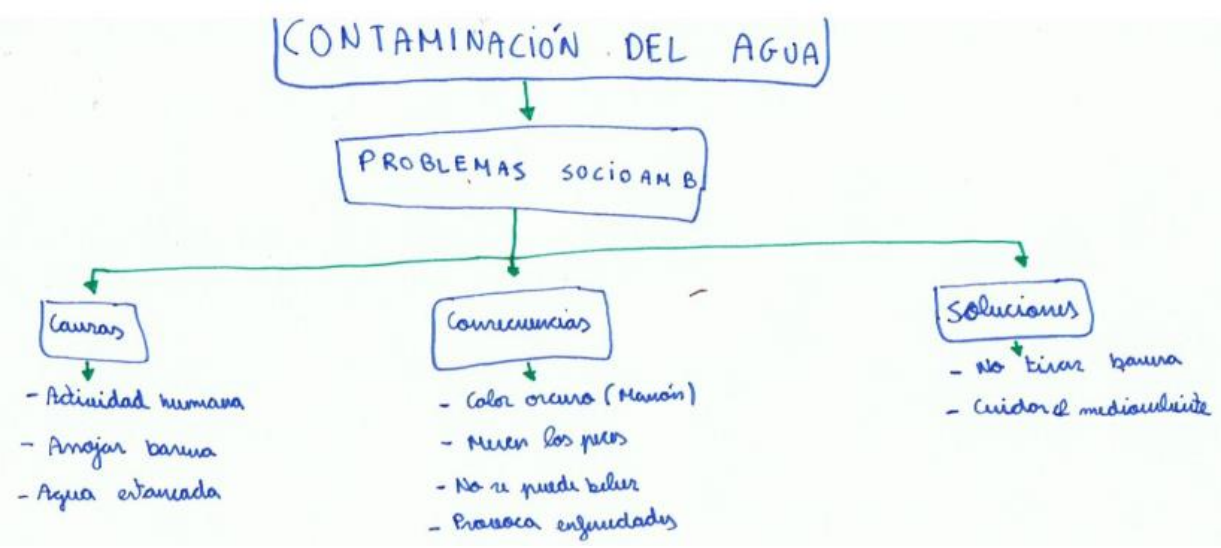

Fuente: Elaborado por estudiantes de la clase $3^{\circ}$ B. IES Catedrático Pulido Rubio, Bonares, Huelva, 2017. 
En este ejemplo se puede visualizar un nivel bajo en conceptos $(<9)$, número de conectores inexistentes (0), un bajo nivel de jerarquía (ya que al no contemplar conectores no se puede dar como válida ninguna proposición. Además, hay proposiciones erróneas) y por último, en relación con el impacto visual, este se presenta poco estructurado.

Figura 2. Ejemplo de un mapa conceptual posterior de nivel medio

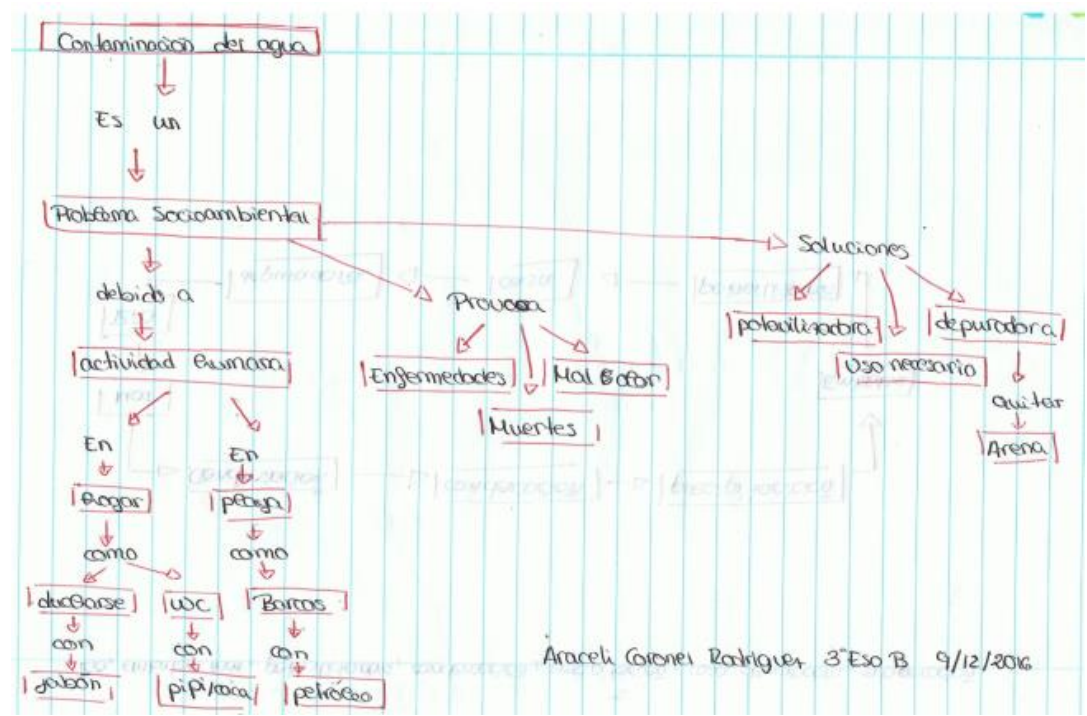

Fuente: Elaborado por estudiantes de la Clase $3^{\circ}$ B. IES Catedrático Pulido Rubio, Bonares, Huelva, 2017.

En este ejemplo de nivel medio se reflejan más de 11 conceptos, más de 10 conectores, un nivel de jerarquía de 3 y una sensación de impacto visual como algo estructutado. Alguna proposición con conectores de nivel medio sería: "La contaminación del agua es un problema socioambiental que provoca enfermedades, muertes y mal olor". En relación con el nivel de jerarquía, se ha considerado de nivel 3, ya que en algunas proposiciones se llega a un mayor nivel de diferenciación progresiva. No obstante, la mayoría de las proposiciones reflejan un menor nivel. 
Figura 3. Ejemplo de un mapa conceptual posterior de nivel alto
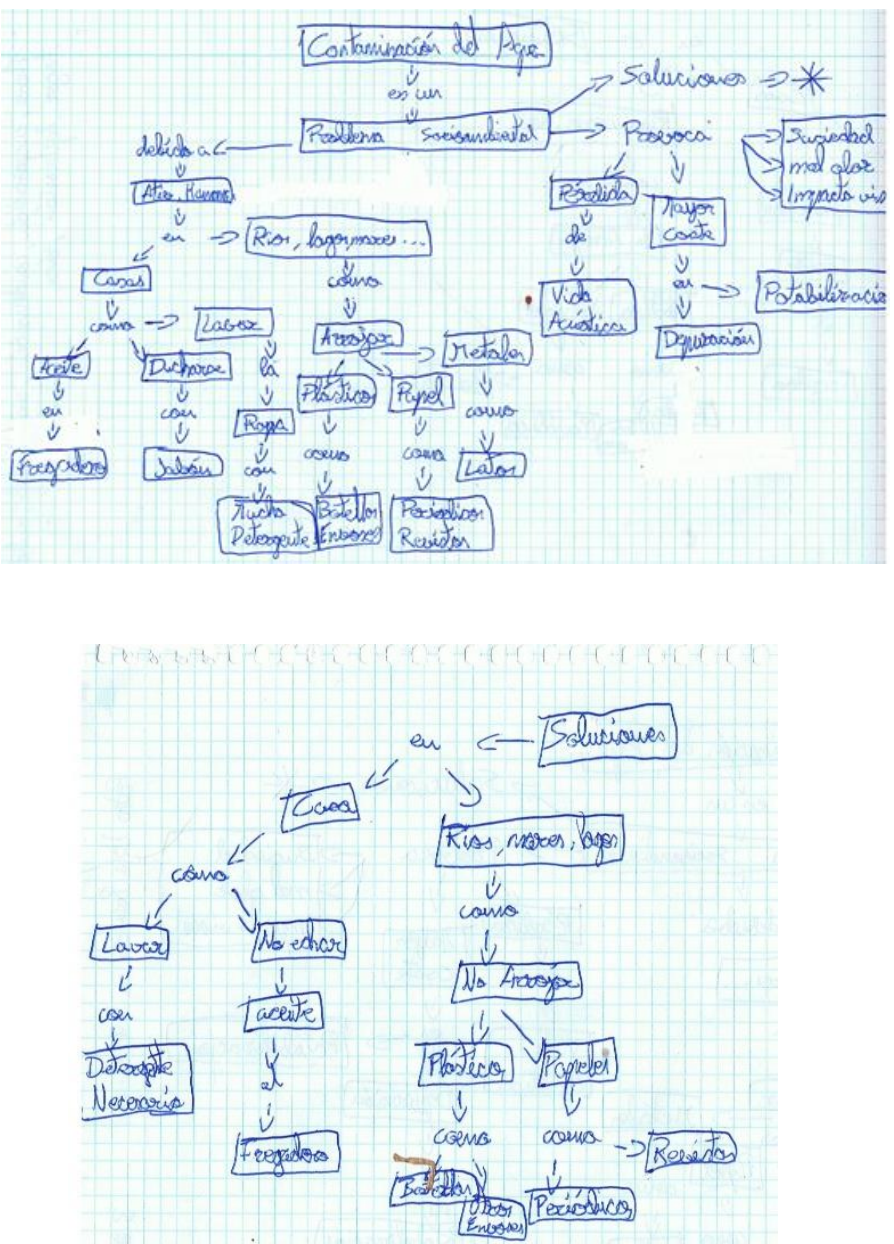

Fuente: Elaborado por estudiantes de la clase $3^{\circ} \mathrm{B}$. IES Catedrático Pulido Rubio, Bonares, Huelva, 2017.

Este mapa conceptual se ha caracterizado en un nivel alto con respecto a los criterios considerados. En este sentido, el número de conceptos es elevado (>21), así como el número de conectores (>31), el nivel de jerarquía (5) y el impacto visual (bastante estructurado). Algunas proposiciones con conectores de nivel alto serían: "la contaminación del agua es un problema socioambiental, debido a la actividad humana en el hogar, como tirar el aceite por el desagüe". Por último, con respecto al nivel de jerarquía, aunque existen niveles de jerarquía de 3 , en la mayoría de las proposiciones se da una buena diferenciación progresiva, incluso se llega al nivel 5.

Otro criterio de evaluación tomado en cuenta ha sido el de las relaciones entre los conceptos, se valora una serie de unidades de significado identificadas en los mapas, las cuales se corresponden con las siguientes dimensiones: causas (las causas que originan la 
contaminación del agua); consecuencias (las consecuencias que provoca la contaminación del agua) y soluciones (las soluciones propuestas para prevenir y disminuir la contaminación). Por otro lado, según la adecuación de las relaciones a las dimensiones de las unidades de significado, se han generado 3 niveles: bajo, medio y alto, tal como puede observarse en la Tabla 2 y en las Figuras 4, 5 y 6.

Tabla 2. Criterio de valoración del primer instrumento de acuerdo con relación entre los conceptos

\begin{tabular}{|l|l|l|l|}
\hline \multicolumn{1}{|c|}{ Dimensiones } & \multicolumn{1}{|c|}{ Nivel bajo } & \multicolumn{1}{c|}{ Nivel medio } & \multicolumn{1}{c|}{ Nivel alto } \\
\hline Causas & $\begin{array}{l}\text { Identifica actividades } \\
\text { de pequeño rango } \\
\text { genéricas y abstractas } \\
\text { con sustancias } \\
\text { contaminantes }\end{array}$ & $\begin{array}{l}\text { Identifica, además, tipo } \\
\text { de actividad de mayor } \\
\text { rango }\end{array}$ & $\begin{array}{l}\text { ldentifica tipo de } \\
\text { actividad de mayor } \\
\text { rango con sus } \\
\text { correspondientes } \\
\text { sustancias } \\
\text { contaminantes }\end{array}$ \\
\hline Consecuencias & $\begin{array}{l}\text { Muestra } \\
\text { consecuencias muy } \\
\text { genéricas y abstractas } \\
(1 \text { o 2) }\end{array}$ & $\begin{array}{l}\text { Muestra } \\
\text { consecuencias muy } \\
\text { genéricas y abstractas } \\
\text { (3 o 4) }\end{array}$ & $\begin{array}{l}\text { Muestra } \\
\text { consecuencias } \\
\text { concretas directas, } \\
\text { identifica a los } \\
\text { elementos que afecta. }\end{array}$ \\
\hline Soluciones & $\begin{array}{l}\text { Señala principalmente } \\
\text { soluciones genéricas e } \\
\text { indirectas (1 o 2) }\end{array}$ & $\begin{array}{l}\text { Señala principalmente } \\
\text { soluciones genéricas e } \\
\text { indirectas (2 o 4) }\end{array}$ & $\begin{array}{l}\text { Señala soluciones } \\
\text { concretas y directas } \\
\text { para abordar el } \\
\text { problema }\end{array}$ \\
\cline { 3 - 4 } & & &
\end{tabular}

Fuente: elaboración propia (Galván Pérez, 2017)

Algunos ejemplos para interpretar dichos criterios, serían:

Tabla 3. Interpretación de criterio de valoración del primer instrumento de acuerdo con relación entre los conceptos

\begin{tabular}{|c|c|c|c|}
\hline Dimensiones & Nivel bajo & Nivel medio & Nivel alto \\
\hline Causas & Tirar basura a la calle & $\begin{array}{l}\text { Lavar la ropa con } \\
\text { detergente }\end{array}$ & $\begin{array}{l}\text { Actividad agrícola con } \\
\text { pesticidas, industrial } \\
\text { con petróleo y } \\
\text { doméstica con jabón }\end{array}$ \\
\hline Consecuencias & Mal olor, suciedad & $\begin{array}{l}\text { Enfermedades, muerte } \\
\text { de los seres vivos, mal } \\
\text { olor, impacto visual, } \\
\text { suciedad, }\end{array}$ & $\begin{array}{l}\text { La pérdida de } \\
\text { biodiversidad acuática, } \\
\text { el desplazamiento de } \\
\text { la fauna terrestre y la } \\
\text { lucha por los recursos, } \\
\text { mayor coste en la } \\
\text { depuración, perjuicio a } \\
\text { la economía pesquera }\end{array}$ \\
\hline Soluciones & $\begin{array}{l}\text { Cuidar el medio } \\
\text { ambiente, reciclar }\end{array}$ & $\begin{array}{l}\text { no arrojar basuras al } \\
\text { mar, no echar aceite al } \\
\text { fregadero, uso } \\
\text { necesario del producto, }\end{array}$ & $\begin{array}{l}\text { Regular y castigar los } \\
\text { vertidos, depurar las } \\
\text { aguas residuales, } \\
\text { utilizar productos } \\
\text { químicos más } \\
\text { naturales }\end{array}$ \\
\hline
\end{tabular}

Fuente: elaboración propia (Galván Pérez, 2017) 
Figura 4. Ejemplo de un mapa conceptual previo de nivel bajo según el criterio de relaciones entre conceptos

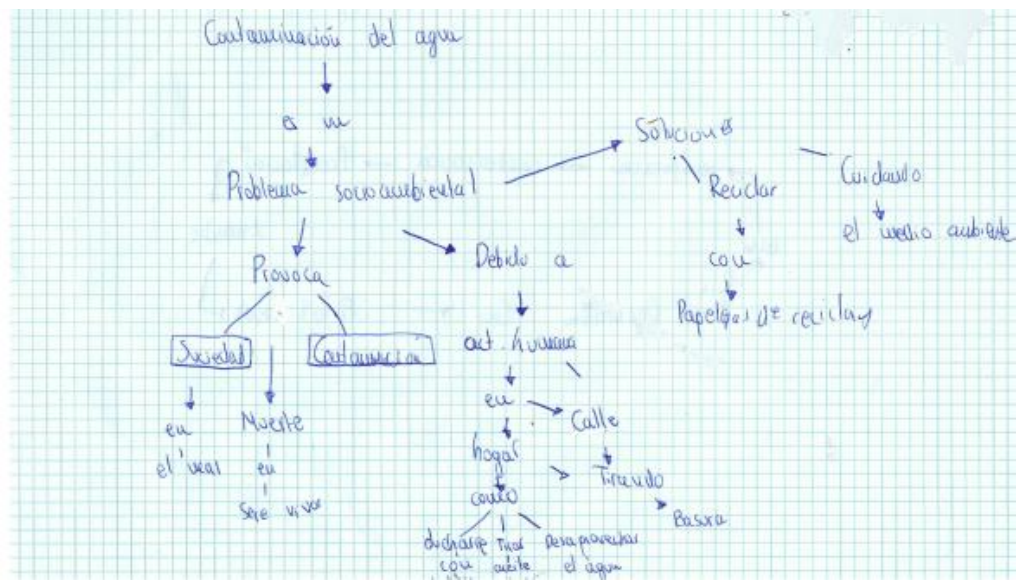

Fuente: Elaborado por estudiantes de la clase $3^{\circ} \mathrm{B}$. IES Catedrático Pulido Rubio, Bonares, Huelva, 2017.

Figura 5. Ejemplo de un mapa conceptual posterior de nivel medio según el criterio de relaciones entre conceptos

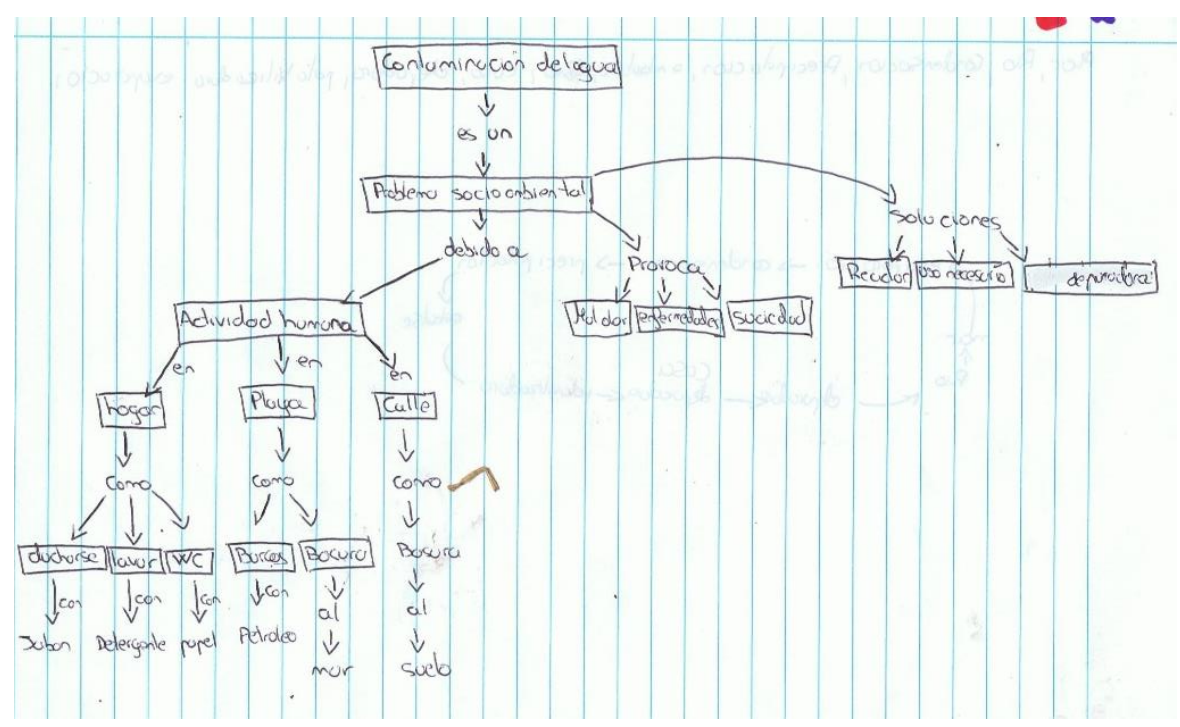

Fuente: Elaborado por estudiantes de la clase $3^{\circ}$ B. IES Catedrático Pulido Rubio, Bonares, Huelva, 2017. 
Figura 6. Ejemplo de un mapa conceptual posterior de nivel alto según el criterio de relaciones entre conceptos

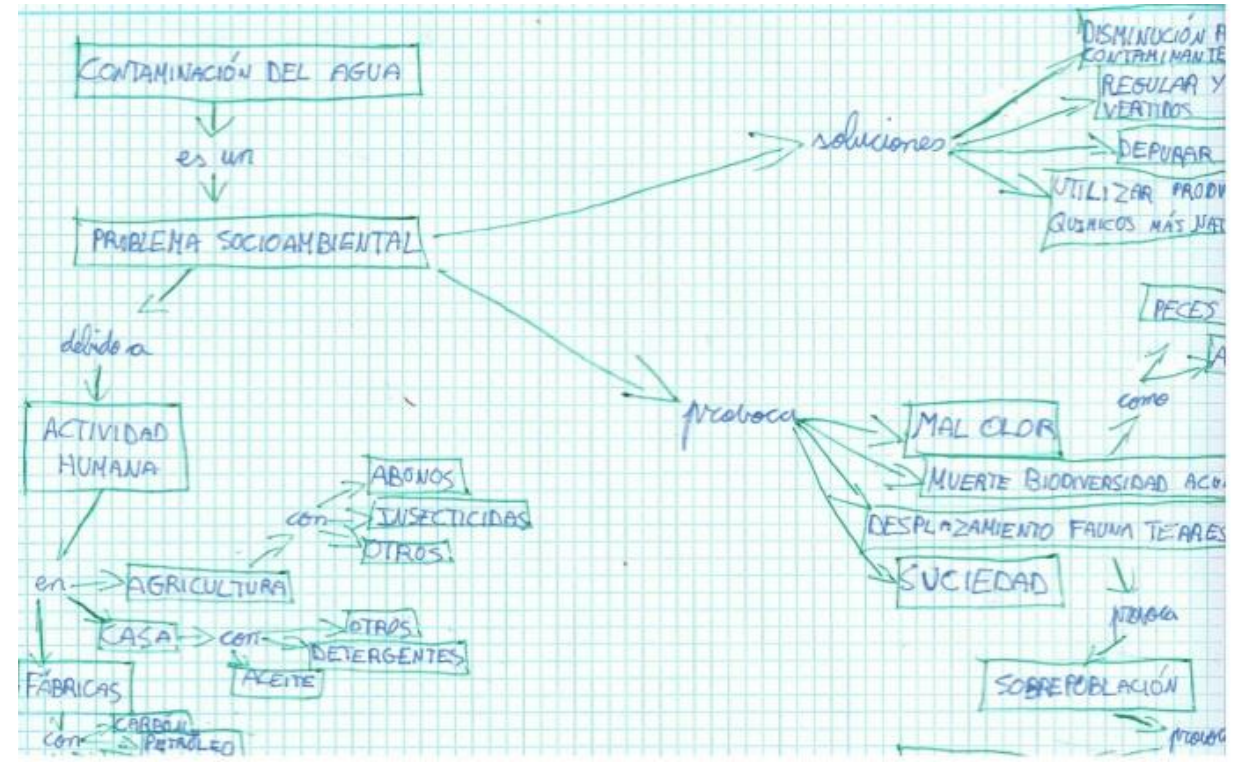

Fuente: Elaborado por estudiantes de la Clase $3^{\circ}$ B. IES Catedrático Pulido Rubio, Bonares, Huelva, 2017.

Con respecto al segundo instrumento de evaluación, este se ha basado en propuestas de estudios anteriores (Miller et al., 2009; Murga-Menoyo, Bautista y Novo, 2011; Proctor y Bernstein, 2013; Pontes y Varo, 2016) tras una búsqueda bibliográfica y documental sobre la evaluación de mapas conceptuales. Esta propuesta permite valorar los mapas conceptuales de 0 a 10 en relación con una serie de variables más flexibles, abiertas y subjetivas, el cual permite obtener 3 niveles de valoración, asociados al nivel de aprendizaje conceptual y actitudinal en torno al agua, como puede observarse en la Tabla 3 y figuras 7,8 y 9 .

Tabla 3. Criterios de valoración del segundo instrumento de evaluación de mapas conceptuales

\begin{tabular}{|c|c|l|}
\hline Nivel de aprendizaje & Puntuación & \multicolumn{1}{|c|}{ Descripción } \\
\hline Nivel bajo & $0-4$ puntos & $\begin{array}{l}\text { El mapa conceptual (MC) muestra ideas confusas o } \\
\text { ausencia de conceptos importantes con pocas } \\
\text { relaciones entre los conceptos con pobre impacto visual }\end{array}$ \\
\hline Nivel medio & $4-7$ puntos & $\begin{array}{l}\text { El MC recoge algunos conceptos importantes, pero } \\
\text { presenta deficiencias de tipo semántico (frases de } \\
\text { enlace inadecuadas, nodos con varios conceptos } \\
\text { diferentes) con alguna jerarquización }\end{array}$ \\
\hline Nivel alto & $7-10$ puntos & $\begin{array}{l}\text { El MC incluye casi todos los conceptos relevantes del } \\
\text { vídeo y los relaciona mediante frases de enlace } \\
\text { adecuadas con buena jerarquización y buen impacto } \\
\text { visual }\end{array}$ \\
\hline
\end{tabular}

Fuente: Elaboración propia (Galván Pérez, 2017) 
Figura 7. Ejemplo de un mapa conceptual previo de nivel bajo de aprendizaje

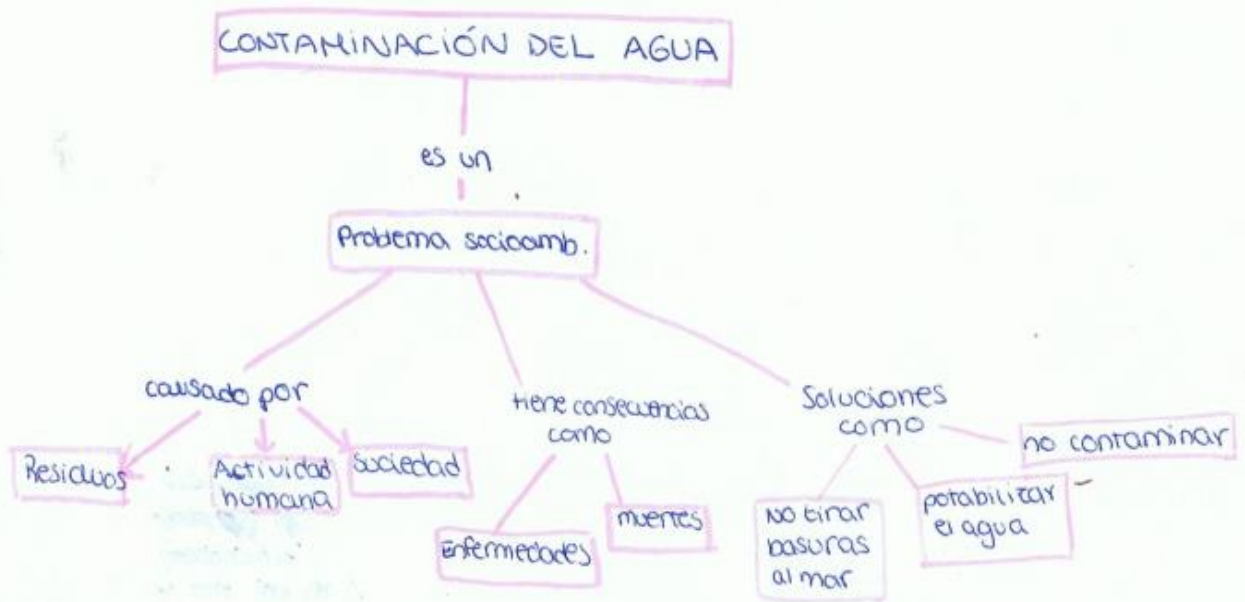

Fuente: Elaborado por estudiantes de la Clase $3^{\circ}$ B. IES Catedrático Pulido Rubio, Bonares, Huelva, 2017.

Figura 8. Ejemplo de un mapa conceptual previo de nivel medio de aprendizaje

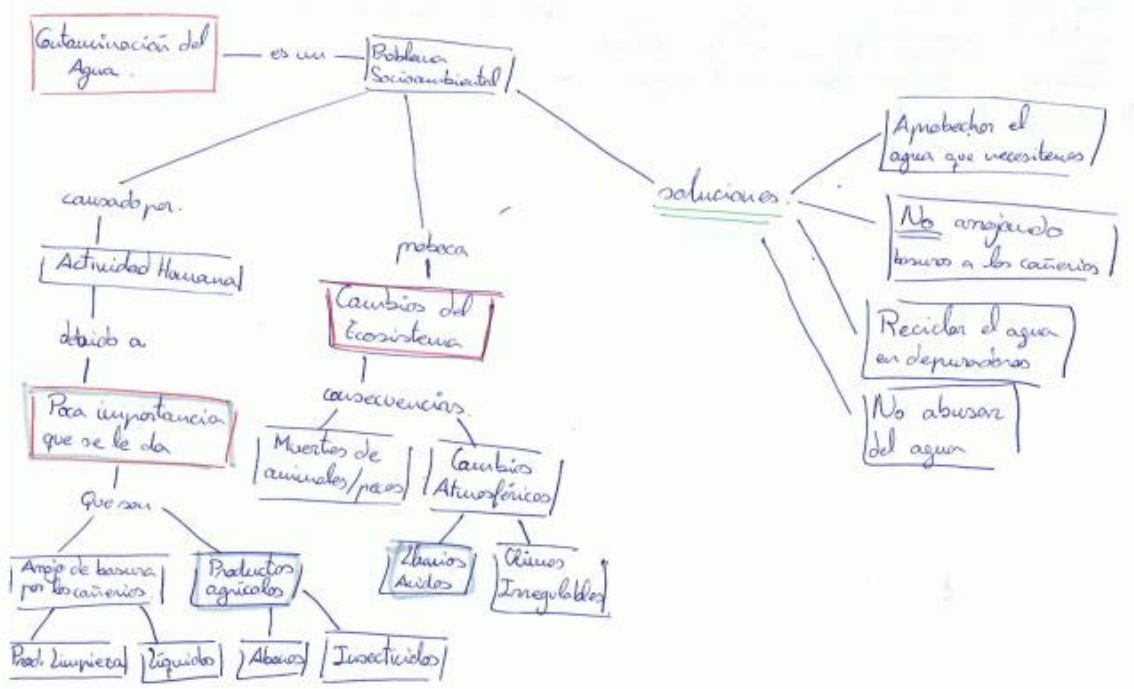

Fuente: Elaborado por estudiantes de la Clase 3ํㅡ. IES Catedrático Pulido Rubio, Bonares, Huelva, 2017. 
Figura 9. Ejemplo de un mapa conceptual posterior de nivel alto de aprendizaje

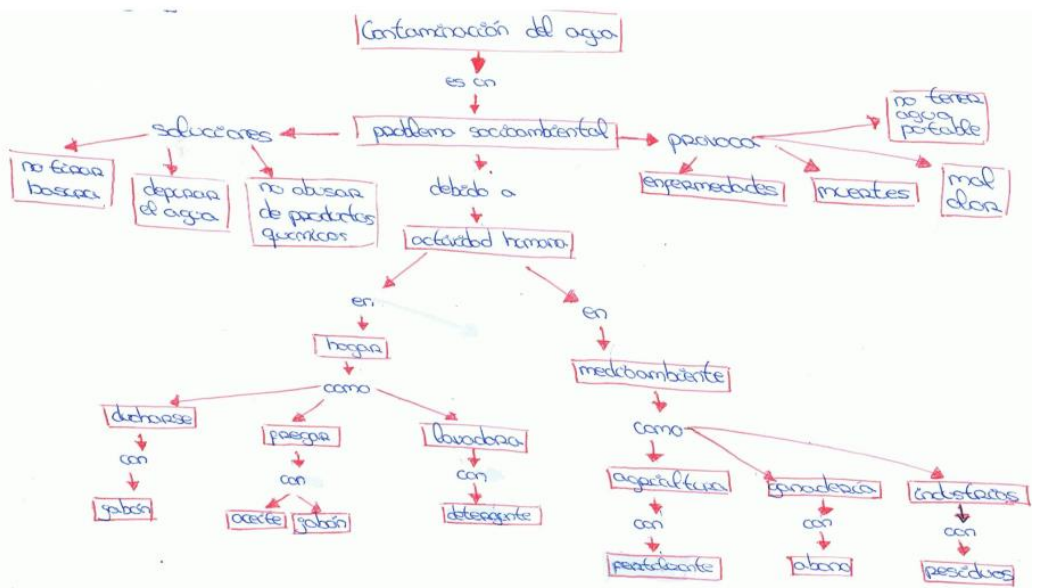

Fuente: Elaborado por estudiantes de la Clase 3ํㅡ. IES Catedrático Pulido Rubio, Bonares, Huelva, 2017.

\section{Resultados}

A continuación, se muestran los resultados obtenidos tras la aplicación de los dos instrumentos de evaluación en los mapas conceptuales elaborados por el alumnado sobre la contaminación del agua, desarrollados en una fase previa de detección de ideas, y en una fase posterior tras la proyección del recurso educativo.

\subsection{Evaluación de mapas conceptuales (PRE)}

En cuanto al instrumento de evaluación por criterios, se observa que, con respecto al número de conceptos (Figura10), número de conectores (Figura 11) y nivel de jerarquización (Figura 12), estos se sitúan principalmente en el nivel bajo: se utilizan de 0 a 10 conceptos, de 0 a 15 conectores y de 1 a 2 en nivel de jerarquía, como puede observarse en las siguientes gráficas. 
Figura 10. Número de mapas conceptuales previos según nivel de criterio de evaluación de los conceptos

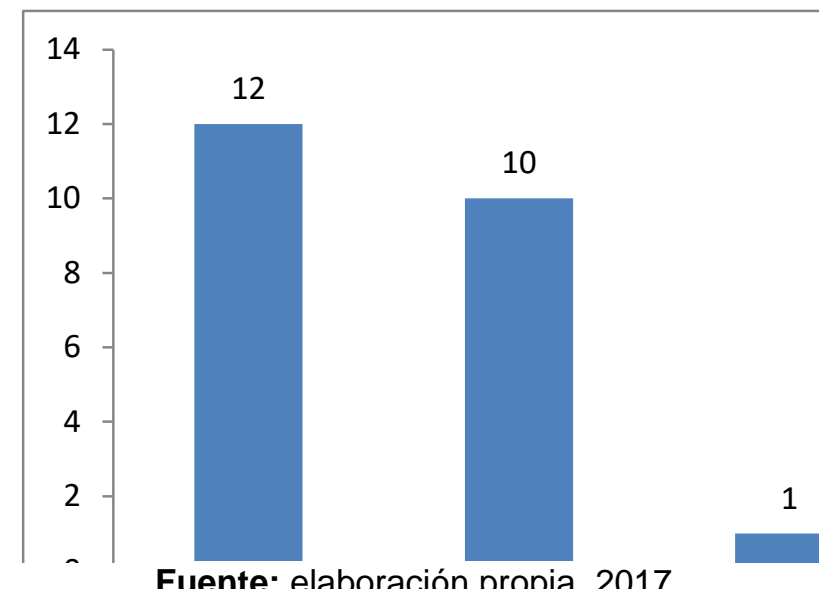

Fuente: elaboración propia, 2017

Figura 11. Número de mapas conceptuales previos según nivel de criterio de evaluación de los conectores

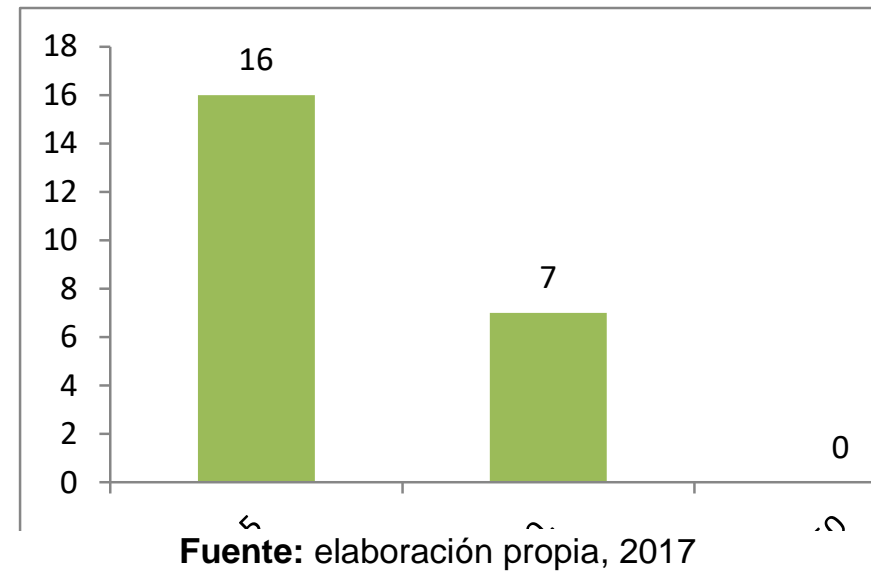

Figura 12. Número de mapas conceptuales previos según nivel de criterio de evaluación de la jerarquía

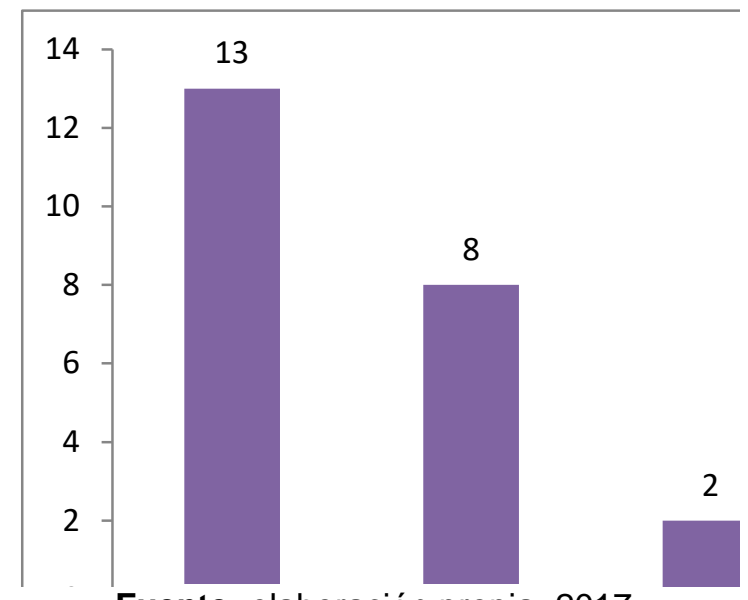

Fuente: elaboración propia, 2017 
Por otro lado, en cuanto al criterio de impacto visual, existe poca diferencia entre el nivel bajo y el nivel medio, donde se coloca mayoritariamente el alumnado, excepto dos personas que son capaces de obtener un nivel alto (ver Figura 13). Por último, si se analiza el criterio de valoración "las relaciones entre los conceptos", se observa que con respecto a las causas y soluciones, se sitúan mayoritariamente en el nivel bajo (con respecto a las causas: identifica actividades de pequeño rango con sustancias contaminantes y con respecto a las soluciones: concreta una o dos de carácter muy genérica y abstracta relacionada con la temática); mientras que las consecuencias se sitúan en un nivel medio (consecuencias muy genéricas y abstractas de 2 a 4), siendo minoritarias aquellas causas, consecuencias y soluciones registradas en un nivel alto (ver Figura 14).

Figura 13. Número de mapas conceptuales previos según nivel de criterio de evaluación del impacto visual

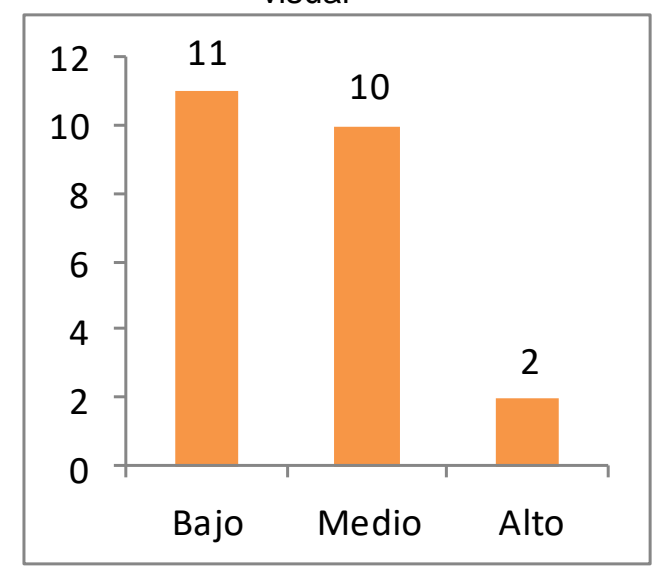

Fuente: Elaboración propia, 2017

Figura 14. Número de mapas conceptuales previos según criterio de evaluación de las relaciones entre los conceptos por nivel

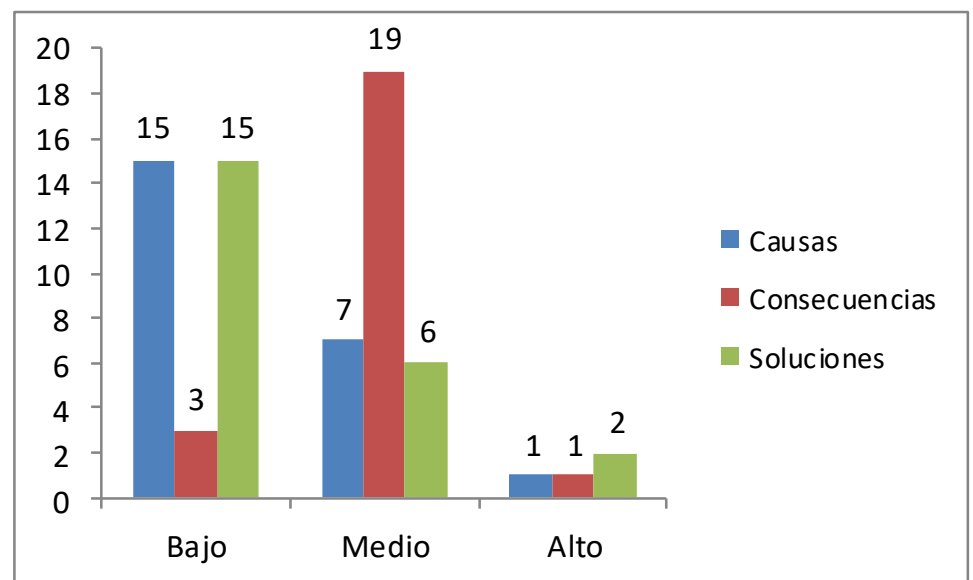

Fuente: Elaboración propia, 2017 
A continuación, en la figura número 15, se muestran los resultados obtenidos aplicando el segundo instrumento de evaluación. Los datos muestran que la mayoría de los mapas conceptuales sobre contaminación del agua, elaborados por el alumnado, se sitúan en un nivel bajo, señalan ideas confusas o ausencia de conceptos importantes, se evidencian pocas relaciones entre los conceptos, pobre impacto visual. Por otro lado, también cabe destacar que en algunos casos se llega alcanzar un nivel medio, detallando algunos conceptos importantes, pero presentando deficiencias de tipo semántico. Finalmente, el nivel alto obtuvo un registro minoritario.

Figura 15. Número de mapas conceptuales previos según nivel de criterio de evaluación del aprendizaje

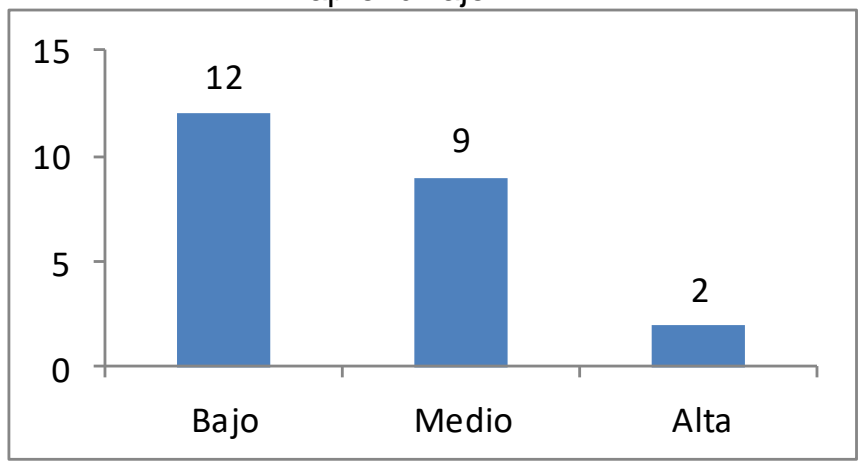

Fuente: Elaboración propia, 2017

Una vez recopilada la información y analizada con los diferentes instrumentos de evaluación de los mapas conceptuales, elaborados por el alumnado, se pueden determinar las ideas previas de los mismos. A continuación, se citan algunos ejemplos tomados de los mapas:

- Se especificaban como causa de la contaminación del agua, mayoritariamente, los términos de "residuos" o "basuras", se identificaron, en mayor medida, residuos o contaminantes sólidos (objetos cotidianos perceptibles a simple vista, como plásticos, latas, etc.) frente a sustancias o contaminantes líquidos o gaseosos (detergentes, petróleo, entre otros). En ningún caso fue citado otro tipo de contaminación del agua, como por ejemplo, la contaminación térmica. Se identificaban como consecuencias, principalmente, el mal olor y la suciedad, y se arrojaban ideas como "ecosistema sucio" o "agua no potable".

- Como solución principal destaca: "no tirar residuos al mar o a la calle". 
- Algunas personas mezclaban las ideas relativas a las causas y a las consecuencias.

- Se confunden los procesos de depuración y potabilización.

- Se daban, también, como soluciones, ideas muy genéricas o simplistas como "limpiar la suciedad del agua", "buscar sitios donde meter el agua sucia”, "cuidar el agua", "cuidar el medio ambiente", "no contaminar" o "no dejar basura en la playa".

- Cuando se habla de contaminación del agua, el alumnado piensa en términos absolutos o globales, llegando a sus máximas consecuencias, sin pensar en término medio, como por ejemplo, cuando el alumnado determina, en muchos casos, las consecuencias de la contaminación como: "agua no potable".

- Solo en muy pocos casos aparecieron términos interesantes, como por ejemplo, "aguas fecales" (causa de contaminación de las aguas), "cambios en los ecosistemas" (como consecuencias de la contaminación del agua) y "vigilar y multar" (como solución a la contaminación del agua).

\subsection{Evaluación de mapas conceptuales (POST)}

Tras la aplicación del instrumento de evaluación, por criterios, en los mapas conceptuales (POST), se observan los siguientes resultados. En relación con los criterios de número de conceptos (Figura 16), número de conectores (Figura 17) y nivel de jerarquía (Figura 18), la valoración de los mapas se sitúa mayoritariamente en un nivel alto, excepto el nivel de jerarquía, que se sitúa principalmente en un nivel medio.

Figura 16. Número de mapas conceptuales previos según nivel de criterio de evaluación de los conceptos

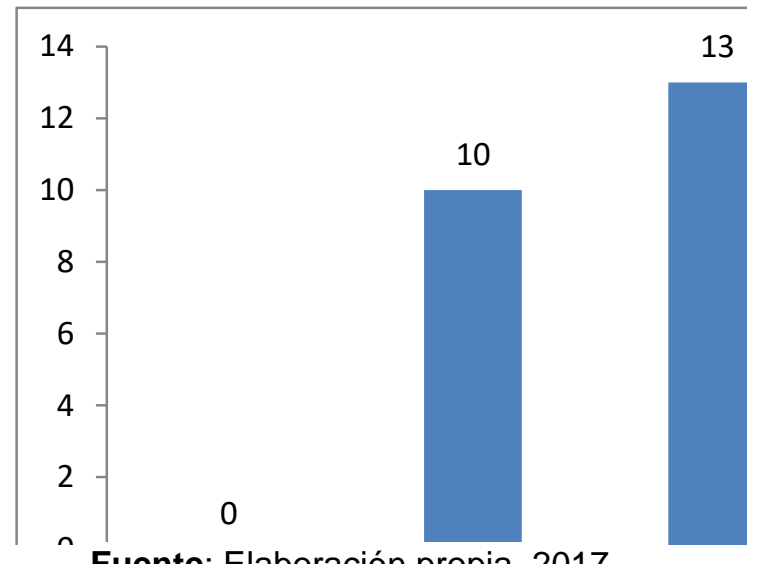

Fuente: Elaboración propia, 2017 
Figura 17. Número de mapas conceptuales previos según nivel de criterio de evaluación de los conectores

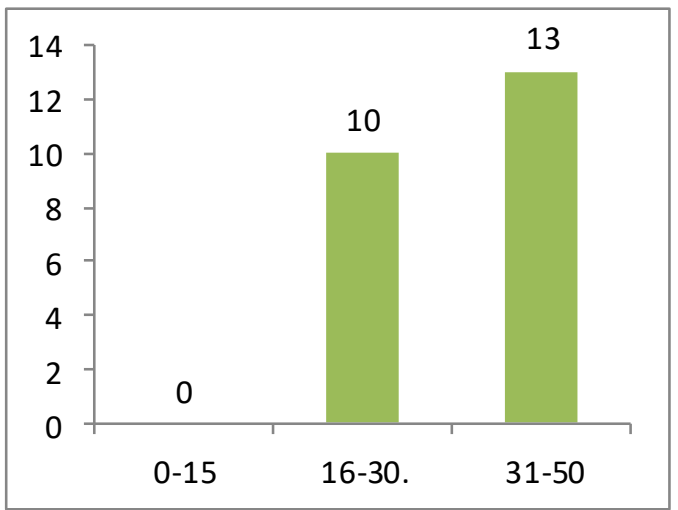

Fuente: Elaboración propia, 2017

Figura 18. Número de mapas conceptuales previos según nivel de criterio de evaluación de la jerarquía

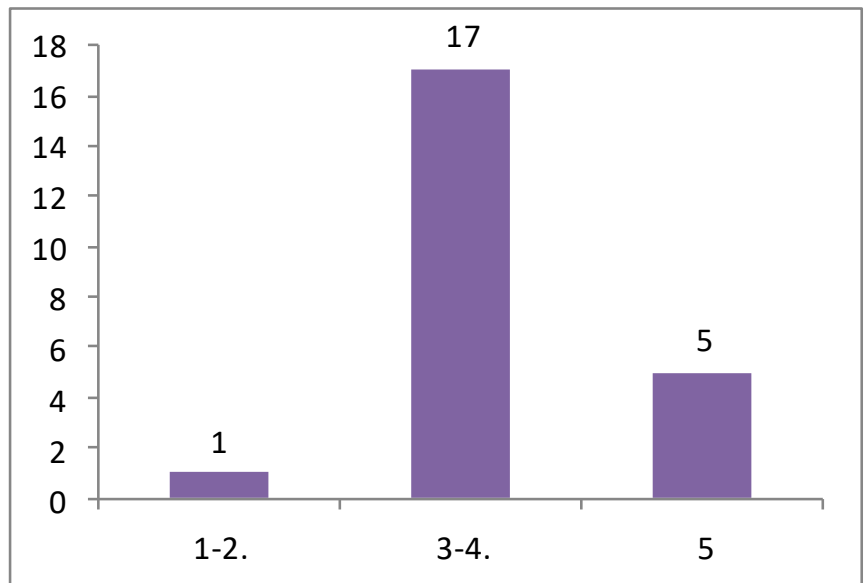

Fuente: Elaboración propia, 2017

Con respecto al impacto visual, principalmente, se alcanza el nivel medio, aunque también se registra una presencia considerable en el nivel alto (ver Figura 19). Por último, en el análisis del criterio "relaciones entre los conceptos" se observa que mayoritariamente se alcanza un nivel medio en las dimensiones de causas, consecuencias y soluciones (Figura 20). 
Figura 19. Número de mapas conceptuales posteriores según nivel de criterio de evaluación del impacto visual

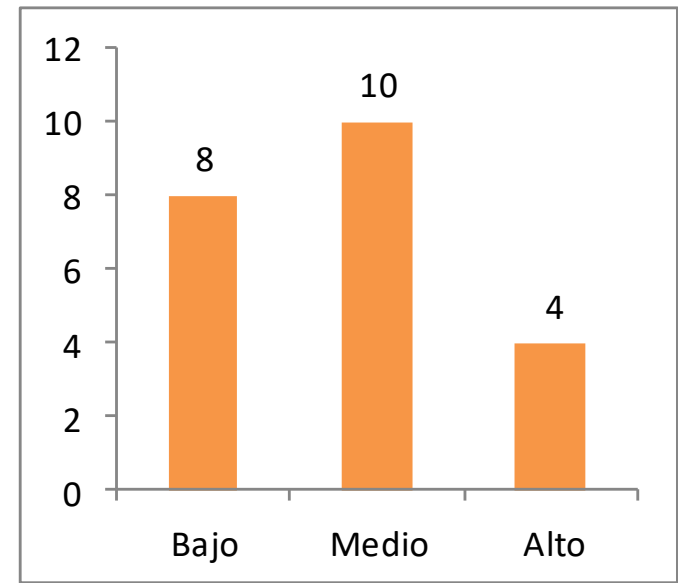

Fuente: Elaboración propia, 2017

Figura 20. Número de mapas conceptuales posteriores según criterio de evaluación de las relaciones entre los conceptos por nivel

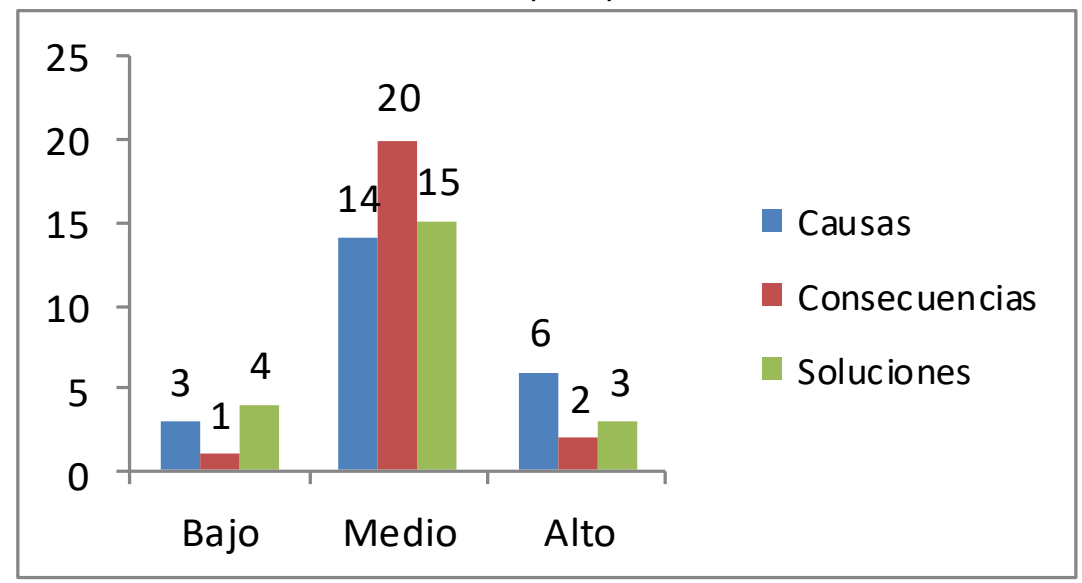

Fuente: Elaboración propia, 2017

A continuación, en la Figura 21, se muestran los resultados obtenidos tras la aplicación del segundo instrumento de evaluación. Dichos resultados reflejan que, principalmente, los mapas conceptuales elaborados por el alumnado alcanzan un nivel alto (se incluyen casi todos los conceptos relevantes y se relacionan mediante frases de enlace adecuadas con buena jerarquización y buen impacto visual), aunque van seguidos muy de cerca por aquellos que se valoran en una calidad media, y muy por encima de aquellos con una calidad baja. 
Figura 21. Número de mapas conceptuales previos según nivel de criterio de evaluación del aprendizaje

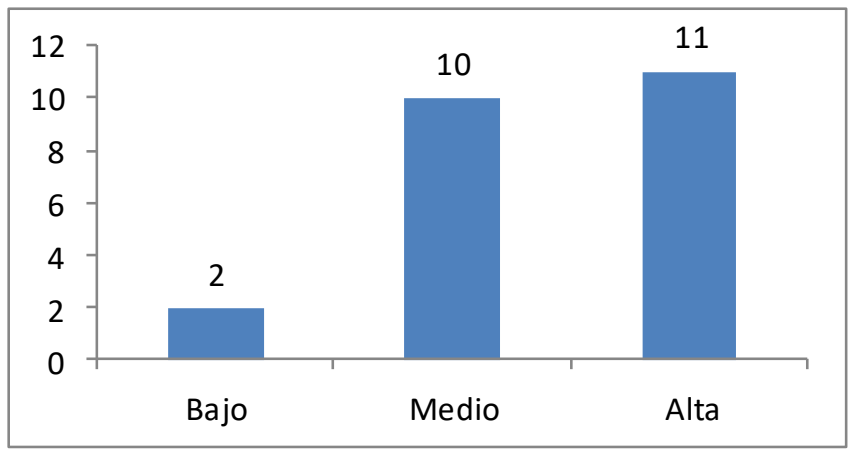

Fuente: Elaboración propia, 2017

\section{Conclusión}

Del análisis de los resultados obtenidos, se puede confirmar, tanto en un análisis pormenorizado como en un análisis global, que el recurso educativo favoreció el aprendizaje conceptual y actitudinal en torno al agua (ver anexo 2. Ejemplos de mapas PRE y POST del alumnado). Como se observa en las gráficas mostradas anteriormente en el apartado de resultados, al realizar una comparativa por cada uno de los criterios de valoración de los mapas conceptuales pre y post, todos y cada uno de ellos aumenta ( $\mathrm{n}^{\circ}$ de conceptos, número de conectores, nivel de jerarquía, impacto visual y las relaciones entre los conceptos).

Tras un análisis comparado de cada criterio se obtendría que, en relación con el "número de conceptos", los mapas conceptuales PRE se sitúan principalmente en un nivel bajo y medio, mientras que los mapas conceptuales POST, tras la aplicación del recurso didáctico y en este mismo criterio, se sitúan principalmente en un nivel medio y alto, siendo además mayoritaria la opción alta, al describir la contaminación del agua entre 21 y 30 conceptos. Estos mismos resultados se obtienen al analizar y comparar el criterio "número de conectores", la opción mayoritaria es aquella que se sitúa en el nivel alto, utiliza una media de 31 a 50 conectores. En cuanto al criterio "nivel de jerarquía", también se pasa de un nivel bajo medio a un nivel medio-alto, siendo esta vez la opción mayoritaria registrada por el alumnado el nivel medio; es decir, se observa en los mapas conceptuales una jerarquía de 3 a 4 niveles. Con respecto al impacto visual, tanto los mapas PRE como POST se sitúan principalmente en un nivel bajo-medio, pero se registra un aumento en el número de mapas conceptuales que llegan alcanzar el nivel alto, concretamente se pasa de 2 a 4 estudiantes. 
Por último, con respecto al último criterio "relaciones entre los conceptos" se registra un avance en las causas y soluciones al pasar principalmente del nivel bajo (en relación a las causas: detalla actividades muy concretas con componentes contaminantes y con respecto a las soluciones: contempla una o dos soluciones muy genéricas y abstractas) al nivel medio (con respecto a las causas: detalla actividades concretas y generales con componentes contaminantes y en relación a las soluciones: se caracteriza por ser genéricas e indirectas, aunque se registran un mayor número de ellas). Llama la atención que la aplicación del recurso educativo no fue capaz de promover un aprendizaje de las consecuencias generadas por la contaminación. Esto resulta evidente, pues la variable se mantiene en un nivel medio tanto en los mapas PRE como en los POST. Lo anterior puede deberse a que el vídeo educativo mostraba consecuencias muy generales y abstractas; por ejemplo: la suciedad, el mal olor, enfermedades y el fin de la vida acuática. Habría sido pertinente complementar la aplicación del vídeo educativo con una ronda de preguntas para que se hiciera hincapié en las consecuencias de la contaminación. Esto habría visibilizado las dudas o contradicciones en torno a dicha cuestión, y con ello se habría alcanzado un mayor avance en la comprensión y aprendizaje.

Finalmente, con los resultados obtenidos mediante el segundo instrumento de evaluación, se observa que, comparativamente, se pasa de un nivel bajo (ideas confusas o ausencia de conceptos importantes y relaciones entre ellos) - medio (presencia de algunos conceptos importantes, pero deficiencias de tipo semántico en los mapas conceptuales PRE) a un nivel medio-alto (incluye casi todos los conceptos relevantes del video y los relaciona mediante frases de enlace adecuadas, con buena jerarquización y buen impacto visual) en los mapas conceptuales POST. En definitiva, se puede afirmar que la aplicación del recurso educativo permitió avanzar en el nivel de aprendizaje conceptual y actitudinal en torno al agua.

En cuanto al cambio actitudinal con respecto a la temática, este se pudo constatar gracias a las anotaciones realizadas en el cuaderno de observación de cada una de las sesiones por el equipo de investigación, así como a la comparativa de dichos elementos de observación con una clase ordinaria (previa entrevista con el profesor tutor). Los resultados arrojaron mayor impacto en cada una de las variables de observación (interés, participación, resolución de dudas, motivación, resolución de la tarea, puntualidad, entre otras). En ese sentido, se podría concluir que el interés hacia la materia favoreció el proceso de aprendizaje 
y despertó la conciencia entre el alumnado acerca de la problemática ambiental que supone la contaminación del agua.

Asimismo, debe destacarse también cómo la aplicación del recurso educativo favoreció el aumento de la concienciación en torno a la temática, hecho que se constata en el registro de las soluciones identificadas en los mapas, como por ejemplo, actitudes de responsabilidad individual: "usar menos productos tóxicos", "aprovechar justo el agua necesaria" y "no utilizar el "wáter" como una papelera". De igual manera se constató en las soluciones colectivas: "vigilar y sancionar los episodios de contaminación", "regular el uso de sustancias tóxicas en los procesos productivos" y "concienciar a la población para disminuir la contaminación".

Finalmente, algunos indicadores observables, que han obstaculizado el avance en el aprendizaje sobre la contaminación del agua, han sido:

- Mayor tiempo disponible para afianzar la metodología de trabajo sobre cómo diseñar mapas conceptuales.

- Dificultad de transformar algunas ideas previas erróneas fuertemente establecidas.

- Grupos más pequeños de estudiantes hubieran facilitado la atención o resolución de dudas.

- La falta de existencia de recursos educativos, en este caso el video, que planteen la contaminación del agua desde un paradigmas más complejo, con alusiones o incorporaciones teóricas en el marco de la nueva cultura del agua y los servicios ecosistémicos.

- Es necesario visibilizar, en mayor medida, diferentes soluciones relacionadas con la contaminación del agua, más allá del ámbito doméstico y privado (buenas prácticas en el hogar), como puede ser el ámbito social (asociacionismo, voluntariado, entre otros), político-municipal (mayor presupuesto, seguimiento y adaptación de procesos de depuración a las localidades en función de su población-equivalente, apertura de procesos participativos en la gestión del agua,...) y económicos (responsabilidad de las industrias de no contaminar, buenas prácticas en las fábricas,...).

\section{Agradecimientos}

El Grupo de Investigación HUM-890, Evaluación en Educación Ambiental, Social e Institucional, del Departamento de Métodos de Investigación y Diagnóstico en Educación de la Facultad de Ciencias de la Educación de la Universidad de Granada se complace en agradecer el tiempo disponible y el esfuerzo desempeñado por el profesorado IES 
Catedrático Pulido Rubio de Bonares (Huelva), por hacer posible la realidad de este proyecto de investigación, así como un agradecimiento también muy especial al alumnado que participó en él.

\section{Referencias}

Araujo, Joaquín. (2012). Agua. Madrid: Gadir.

Ausubel, David, Novak, Joseph y Hanesian, Helen. (1989). Psicología Educativa. México: Trillas.

Ausubel, David. (1968). Educational psychology: a cognitive view. New York: Rinechart \& winston.

Ballester, Antoni. (2002). El aprendizaje significativo en la práctica. Cómo hacer el aprendizaje significativo en el aula. [versión digital pdf]. Recuperado de http://www.aprendizajesignificativo.es/mats/El aprendizaje significativo en la practica .pdf

Bronfenbrenner, Urie. (1987). La ecología del desarrollo humano. Barcelona: Paidós.

Castaño, Enith. (Octubre, 2010). La construcción de mapas conceptuales para fortalecer procesos de autonomía en el aprendizaje. Ponencia presentada en Conference on concept Maps: Making Learning Meaningful, Viña del Mar, Chile. Recuperado de http://cmc.ihmc.us/cmc2010papers/cmc2010-88.pdf

Correia, Paulo, Silva, Amanda y Junior Jerson. (2010). Concept maps as a tool for evaluation in classroom. Revista Brasileira de Ensino de Física, 32(4), 4402-1, 4402-8. Recuperado http://www.scielo.br/pdf/rbef/v32n4/09.pdf

Echarri, Fernado y Puig, Jordi. (2008). Aplicaciones didácticas del Museo de Ciencias Naturales de la Universidad de Navarra: Educación Ambiental y aprendizaje significativo. Revista Seguridad y Medio Ambiente, (112), 28-48. Recuperado de https://www.fundacionmapfre.org/documentacion/publico/i18n/catalogo imagenes/grup o.cmd?path $=1039311$

Fernández, Rosario y Rodríguez, Luis María. (1995). Los mapas conceptuales como instrumento de evaluación, análisis de una experiencia en el área de ciencias. Revista de Educación, (307), 367-379.

Fernández, Jorge y Solís, Emilio. (2011). El agua como recurso para investigar en el aula. Una investigación en la asignatura de Ciencias para el Mundo Contemporáneo. Revista Investigación en el aula, (75), 49-61.

Fracchia, Carina, Armiño, Ana y Martins, Adair. (2015). Realidad aumentada aplicada a la enseñanza de Ciencias Naturales. Revista Iberoamericana de Tecnología en Educación y Educación en Tecnología, (16), 7-15. Recuperado de http://teyetrevista.info.unlp.edu.ar/wp-content/uploads/2016/06/TEYET16-art01.pdf 
Galván, Laura y Gutiérrez, José. (septiembre, 2017). Evaluación de recursos educativoambientales y herramientas de sensibilización y aprendizaje conceptual sobre el agua. Ponencia presentada en el X Congreso Internacional sobre Investigación en Didáctica de la Ciencia, Sevilla, España.

González, Fermín. (2008). El mapa conceptual y el diagrama UVE. Recursos para la Enseñanza Superior en el siglo XXI. Editorial: Narcea.

González, Fermín, Morón, Ciriaco y Novak, Joseph Donald. (2001). Errores conceptuales. Diagnosis, tratamiento, y reflexiones. Pamplona: Eunate.

Gowin, Bob. (1981). Educating. New York: Ithaca.

Guruceaga, Arantzazu y González, Fermín. (2004). Aprendizaje significativo y educación ambiental: análisis de los resultados de una práctica fundamentada teóricamente. Enseñanza de las Ciencias, 22(1), 115-136. Recuperado de http://www.raco.cat/index.php/ensenanza/article/viewFile/21965/21799

Marcén, Carmelo. (Abril, 2006). El aprendizaje de las ideas de los escolares sobre el agua no surge porque sí. Ponencia en III Jornadas de Educación Ambiental de la Comunidad Autónoma de Aragón, Zaragoza, España.

Martínez, Francisco Javier y Antoranz, María Antonia. (noviembre, 2002). El agua y el sistema educativo español. Ponencia en III Congreso Ibérico sobre Gestión y Planificación del Agua, Sevilla, España.

Miller, Kevin, Koury, Kevin, Fitzgeral, Gail, Hollingsead, Candice, Mitchem, Katherine, Tsai Hsien, Park, Meeaeng. (2009). Concept Mapping as a Research Tool to Evaluate Conceptual Change Related to Instructional Methods. Teacher Education and Special Education, 32(4), 365-378.

Molina-Azcárate, Ladislada. (2013). Los mapas conceptuales como herramientas de diagnóstico y tratamiento de errores conceptuales. Journal for Educators, Teachers and Trainers, 4(1), $122-131$.

López, Marta y Morcillo, Juan Gabriel. (2007) Las TIC en la enseñanza de la Biología en la educación secundaria: los laboratorios virtuales. REEC: Revista electrónica de enseñanza de las ciencias, 6(3), 562-576. Recuperado de http://www.docenciauniversitaria.org/volumenes/volumen6/ART5 Vol6 N3.pdf

Moreno, Gabriel. (2014). El potencial de los vídeos educativos. Revista de Investigación, $81(38), 57-74$.

Murga-Menoyo, María Ángeles, Bautista, María José y Novo, María. (2011). Mapas conceptuales con Cmap Tools en la enseñanza universitaria de la educación ambiental. Estudio de caso en la UNED. Enseñanza de las Ciencias, 29(1), 047-060. Recuperado de http://www.raco.cat/index.php/Ensenanza/article/view/243822/353425

Novak, Joseph Donald. (1977). A Theory of Education. Ithaca, N.Y.: Cornell University Press.

Novak, Joseph Donald. (1978). A Theory of Educaction as a Basis for Environmental Education. En Trilochan S. Bakshi y Zev Naveh (Eds.), Environmental Education. Principles, Methods and applications (pp. 129-138). Nueva York: Plenum Press. 
Novak, Joseph Donald y Gowin, Bob. (1988) Aprendiendo a aprender. Barcelona: Martínez Roca.

Novak, Joseph Donald. (1990). Teoría y práctica de la educación (3ª ed.). Madrid: Alianza Universidad.

Novak, Joseph Donald. (1998). Conocimiento y aprendizaje. Los mapas conceptuales como herramientas facilitadoras para escuelas y empresas. Madrid: Alianza Editorial.

Ojeda, Fernando, Gutiérrez, José y Perales, Francisco Javier. (2011). Enseñanza de las ciencias. Diseño, fundamentación y validación de un programa virtual colaborativo en educación ambiental. Revista de investigación y experiencias didácticas, 1(29), 127146, doi: https://doi.org/10.5565/rev/ec/v29n1.458

Pontes, Alfonso. (2012). Representación y comunicación del conocimiento con mapas conceptuales en la formación del profesorado de ciencia y tecnología. Revista Eureka sobre Enseñanza y Divulgación de las Ciencias, 9(1), 108-125.

Pontes, Alfonso, Serrano, Rocío y Muñoz, Juan Manuel. (2015). Los mapas conceptuales como recurso de interés para la formación inicial del profesorado de enseñanza secundaria: Opiniones del alumnado de ciencias sociales y humanidades. Educación XX1, 18(1), 99-124. doi: 10.5944/educXX1.18.1.12313

Pontes, Alfonso y Varo, Marta. (2016). Mapas conceptuales aplicados al tratamiento de temas medioambientales en la formación del profesorado de física. Revista Currículum y formación al profesorado, 20(2), 452-472. Recuperado de http://www.redalyc.org/pdf/567/56746946025.pdf

Pozo, Juan Ignacio. (1989). Teorías cognitivas del aprendizaje. Madrid: Ed. Morata.

Prieto, Ana Beatriz y Chrobak, Ricardo. (2013). Integración de TICs, investigación y herramientas metacognitivas en la educación de ciencias y ambiental. Estudio de caso: disponibilidad de agua de las cuencas del noroeste de Patagonia y su relación con la actividad solar. Journal for Educators, Teachers and Trainers, 4(1), 132-141. Recuperado de http://digibug.ugr.es/handle/10481/28126\#.WjfsoTdG3cs

Proctor, James y Bernstein, Jennifer. (2013). Environmental Connections and Concept Mapping: Implementing a New Learning Technology at Lewis \& Clark College. Journal of Environmental Studies and Sciences, 38(1), 30-41. Recuperado de https://college.Iclark.edu/live/files/13576-proctorbernstein2013pdf

Rivadulla, Juan, García Barros, Cristina y Martínez, Cristina. (2015). Los mapas conceptuales como instrumentos para analizar las ideas de los estudiantes de Maestro de Educación Primaria sobre qué enseñar de nutrición en Educación Primaria. Revista Complutense de Educación, 3(2), 1247-1269. Recuperado de https://revistas.ucm.es/index.php/RCED/article/viewFile/47704/48833

Sabbatini, Marcelo. (2004). Centros de ciencia y museos científicos virtuales: teoría y práctica (Tesis doctoral) Universidad de Salamanca, España. Recuperado de http://www.sabbatini.com/marcelo/artigos/tesis-sabbatini.pdf

Valadares, José. (2013). Concept maps and the meaningful learning of science. Journal for Educators, Teachers and Trainers, 4, 180-194. Recuperado de https://www.sciencedirect.com/science/article/pii/S0260691703001205 


\section{Anexos}

\section{Anexo 1. Ficha de trabajo}

FICHA VIDEO "LA NECESIDAD DE DEPURAR"

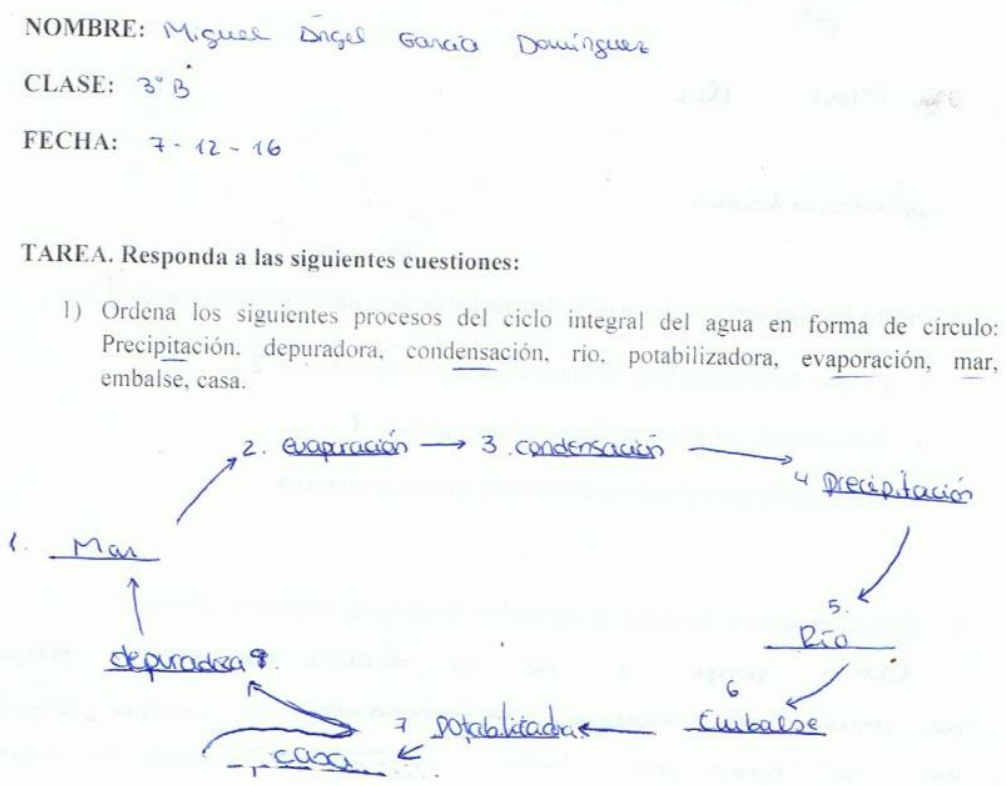

2) Escribe tres tipos de actividades genéricas que generan contaminación del agua

a) Erlian ea aceit por el desagiie del grifo

b) Arrquar basuna por e váter

c) Echar demasian detergente an ece ropa sin recesidad (luvadora)

3) Escribe tres sustancias o productos que contaminan el agua

a) Detergente

b) Aceite

c) Bastonciflos 
4) Escribe tres consecuencias o problemas que genera la contaminación del agua

a) Muertic de peces

b) Mai olor

c) Eufoutdodes

5) Ordena los siguientes procesos de la depuración de las aguas residuales, pon del I al 3

a. Proceso de desinfección ( eliminación de agentes patógenos) 2

b. Petratamiento (eliminan residuos, arenas y grasas) 1

c. Proceso biológico (bacterias eliminan la materia orgánica) 3

6) ¿Consideras que es importante la depuración de las aguas residuales? ¿Por qué?

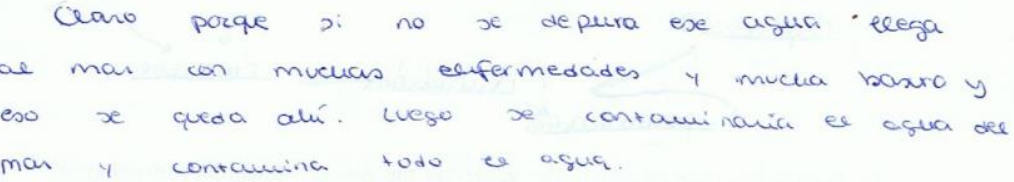

7) ¿Qué tres acciones pueden llevarse en casa para reducir la contaminación y el ahorro del agua? ¿Y fuera de casa?

a) Ecuar en ciceitt en un bote y letarla a in punto
Rempio cercamo.

b) Echar ees residuos sobre ea baxura y no al váter

c) Echear en detergente necesario en ec cavadora. 
Anexo 2. Ejemplos de mapas conceptuales previos y posteriores del alumnado

Ejemplo 1. Mapa conceptual elaborado por alumna, mapa previo (arriba) y mapa posterior (abajo)

$L$
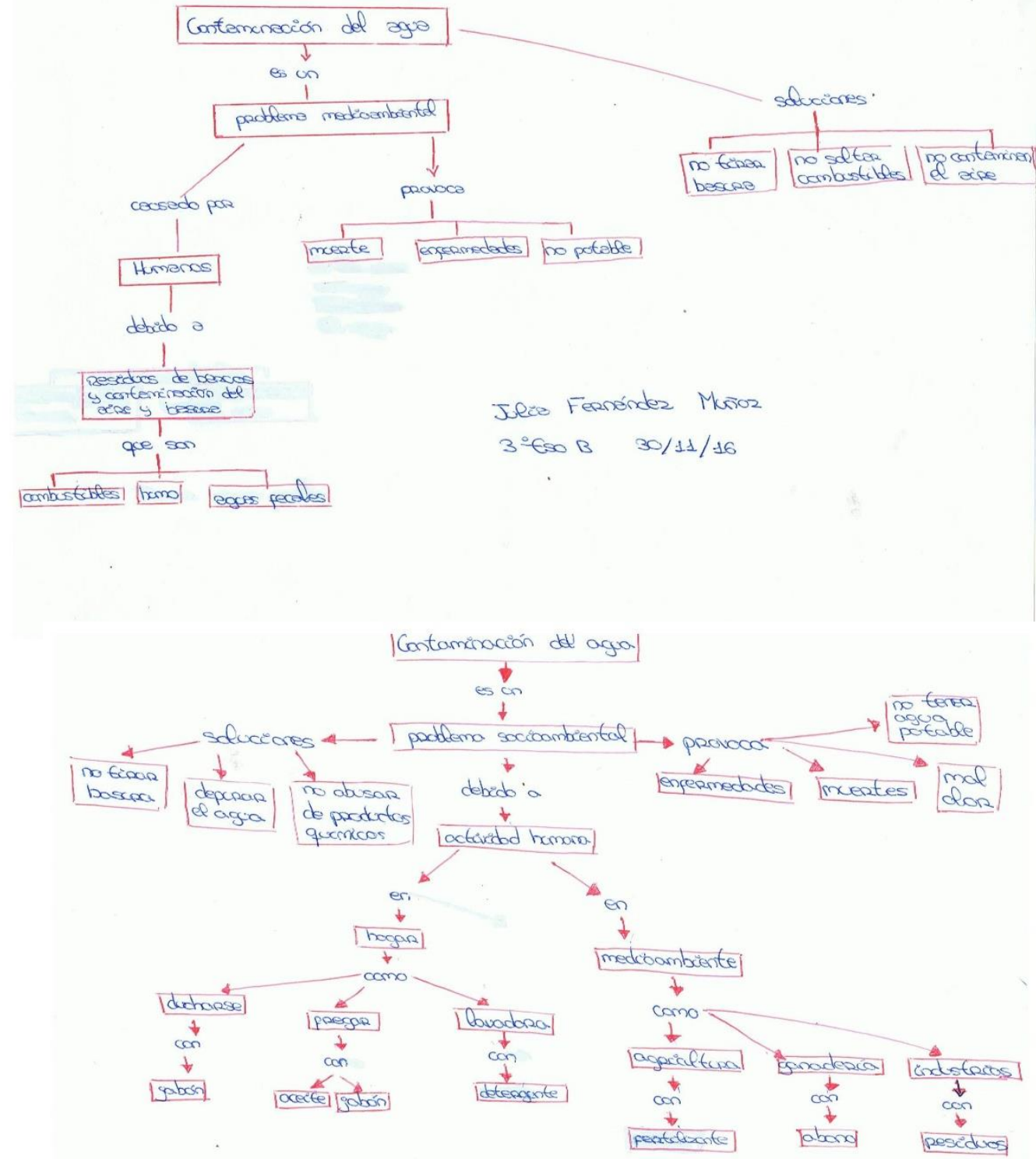
Ejemplo 2. Mapa conceptual elaborado por alumna, mapa previo (arriba) y mapa posterior (abajo)
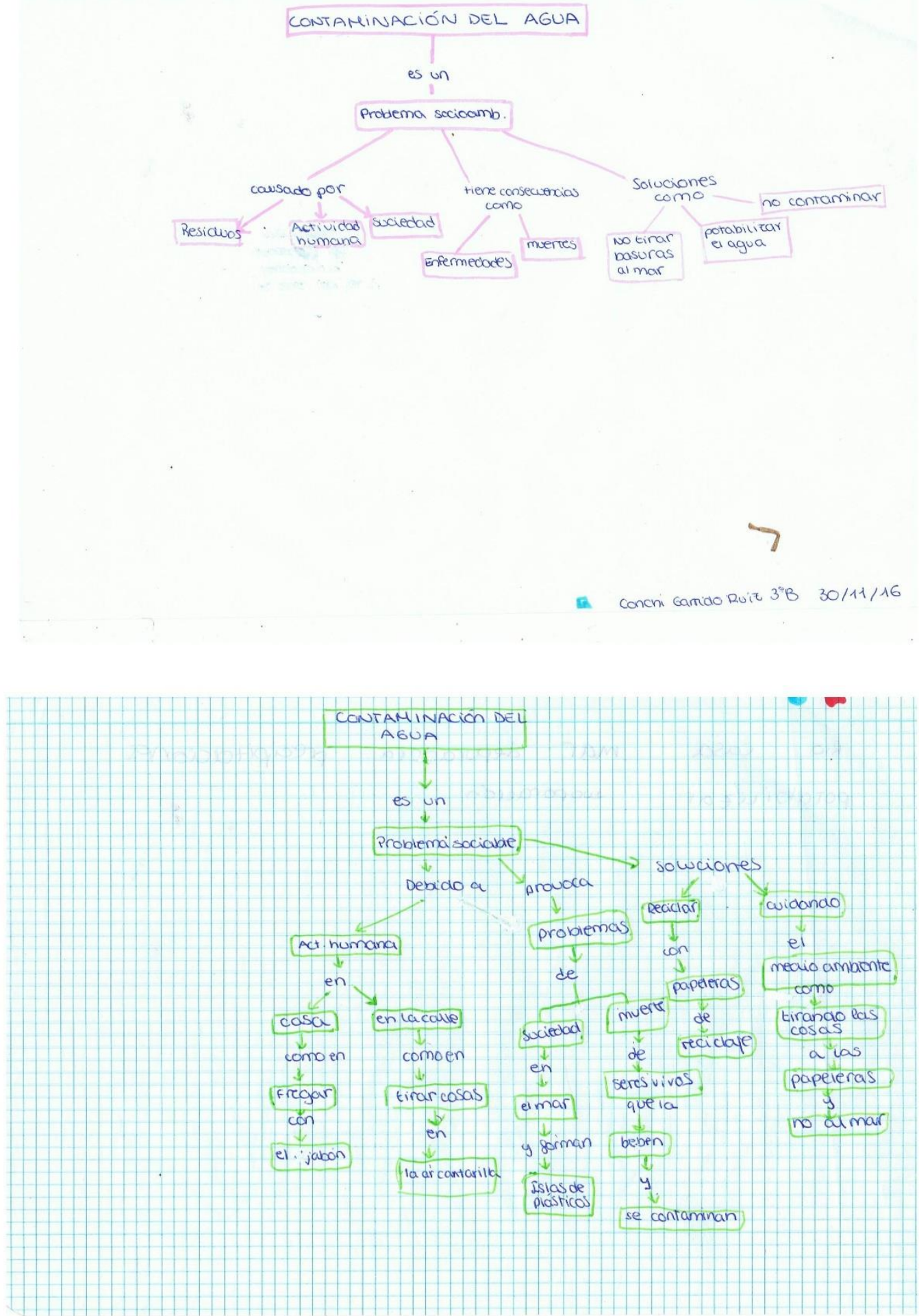


\section{Ejemplo 3. Mapa conceptual elaborado por alumna, mapa previo (arriba) y mapa posterior (abajo)}
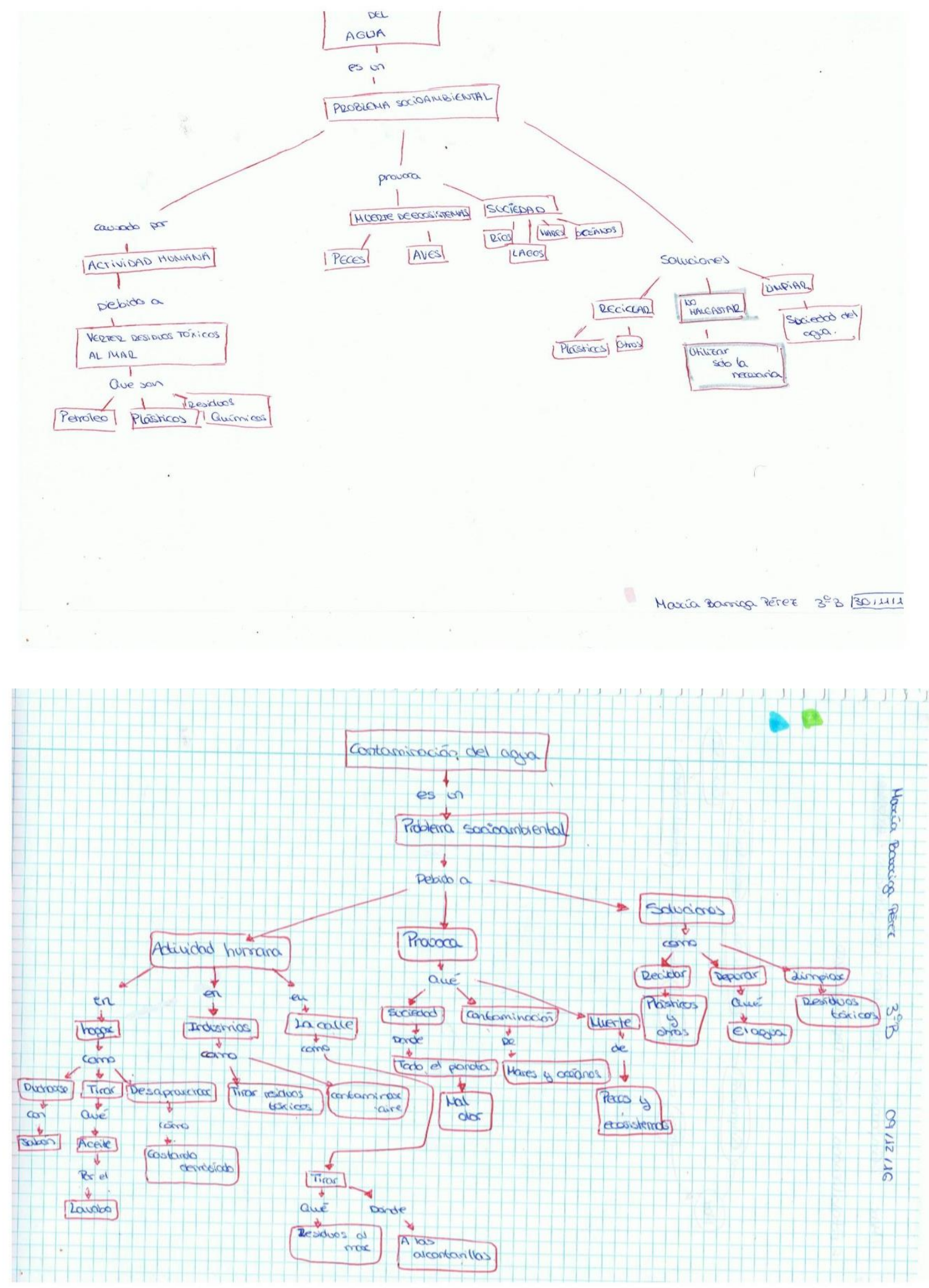

Volumen 18 Número 1, Año 2018, ISSN 1409-4703 